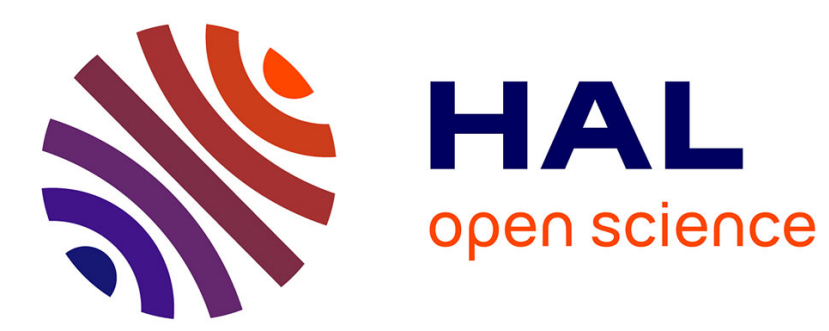

\title{
REILLY-TYPE INEQUALITIES FOR PANEITZ AND STEKLOV EIGENVALUES
}

\author{
Julien Roth
}

\section{To cite this version:}

Julien Roth. REILLY-TYPE INEQUALITIES FOR PANEITZ AND STEKLOV EIGENVALUES. 2017. hal-01539128

\author{
HAL Id: hal-01539128 \\ https://hal.science/hal-01539128
}

Preprint submitted on 14 Jun 2017

HAL is a multi-disciplinary open access archive for the deposit and dissemination of scientific research documents, whether they are published or not. The documents may come from teaching and research institutions in France or abroad, or from public or private research centers.
L'archive ouverte pluridisciplinaire HAL, est destinée au dépôt et à la diffusion de documents scientifiques de niveau recherche, publiés ou non, émanant des établissements d'enseignement et de recherche français ou étrangers, des laboratoires publics ou privés. 


\title{
REILLY-TYPE INEQUALITIES FOR PANEITZ AND STEKLOV EIGENVALUES
}

\author{
JULIEN ROTH
}

\begin{abstract}
We prove Reilly-type upper bounds for different types of eigenvalue problems on submanifolds of Euclidean spaces with density. This includes the eigenvalues of Panetiz-like operators as well as three types of generalized Steklov problems. In the case without density, the equality cases are discussed and we prove some stability results for hypersurfaces which derive from a general pinching result about the moment of inertia.
\end{abstract}

\section{INTRODUCTION}

Let $\left(M^{n}, g\right)$ be a $n$-dimensional $(n \geqslant 2)$ closed, connected, oriented manifold, isometrically immersed by $X$ into the $(n+1)$-dimensional Euclidean space $\mathbb{R}^{n+1}$. The spectrum of the Laplacian of $(M, g)$ is an increasing sequence of real eigenvalues

$$
0=\lambda_{0}(M)<\lambda_{1}(M) \leqslant \lambda_{2}(M) \leqslant \cdots \leqslant \lambda_{k}(M) \leqslant \cdots \longrightarrow+\infty,
$$

counted without multiplicity. The eigenvalue 0 (corresponding to constant functions) is simple and $\lambda_{1}(M)$ is the first positive eigenvalue. In [29, Reilly proved the following well-known upper bound for $\lambda_{1}(M)$

$$
\lambda_{1}(M) \leqslant \frac{n}{V(M)} \int_{M} H^{2} d v_{g}
$$

where $H$ is the mean curvature of the immersion. In the same paper, he also proved an analogous inequality involving the higher order mean curvatures. Namely, for $r \in\{1, \cdots, n\}$

$$
\lambda_{1}(M)\left(\int_{M} H_{r-1} d v_{g}\right)^{2} \leqslant V(M) \int_{M} H_{r}^{2} d v_{g},
$$

where $H_{k}$ is the $k$-th mean curvature, defined as the $k$-th symmetric polynomial of the principal curvatures. Moreover, Reilly studied the equality cases and proved that equality in (1) as in (2) is attained if and only if $X(M)$ is a geodesic sphere. These inequalities have been generalized for other ambient spaces [18, 20], other operators, in particular of Jacobi type [1, 4, 7], in the anisoptropic setting [32] or for weighted ambient spaces $[8,17,33$. In particular, in [33, we prove the following general inequality

$$
\lambda_{1}\left(L_{T, f}\right)\left(\int_{M} \operatorname{tr}(S) \mu_{f}\right)^{2} \leqslant\left(\int_{M} \operatorname{tr}(T) \mu_{f}\right) \int_{M}\left(\left\|H_{s}\right\|^{2}+\|S \nabla f\|^{2}\right) \mu_{f},
$$

where $\mu_{f}=e^{-f} d v_{g}$ is the weighted measure of $(M, g)$ endowed with the density $e^{-f}, T, S$ are two symmetric, free-divergence $(1,1)$-tensors with $T$ positive definite. 
Moreover, $L_{T, f}$ is the second order differential operator defined for any smooth function $u$ on $M$ by

$$
L_{T, f}=-\operatorname{div}(T \nabla u)+\langle\nabla f, T \nabla u\rangle .
$$

In this paper, we are interested in a closely related problems, First, we prove eigenvalue estimates for the Steklov problem for submanifolds with boundary. We obtain general Reilly-type upper bounds (see Theorem 3.1) that generalize the earlier estimates by Ilias and Makhoul [25. These upper bounds are given for a larger class of a general Steklov problem, also involving a weighted measure.

We also consider two other Steklov-type problems, namely the Wentzell-Steklov problem as well as a biharmonic Steklov problem, both in the non-weighted context. Here again, we upper bounds for the first eigenvalues of these problems are given and the equality cases are characterized (see Theorem 3.3 and 3.4 .

Finally, we also prove an upper bound for the first eigenvalue of weighted Paneitz-like operators. These Reilly-type estimates extend the previous estimates by Chen and Li [13. The equality case is also discussed (see Theorems 4.1 and 4.4).

For all the above eigenvalue problems, we derive estimates for submanifolds of spheres and projectives spaces by the use of the standard embeddings, as well as isoperimetric estimates for the case of embedded hypersurfaces. These estimates will be given in Section 5

The last part of the present paper is devoted to stability results. As mentionned, the equality cases are discussed and we can obtain for the case of hypersurfaces some stability results with proximity to geodesic hyperspheres in case of almost equality. Precisely, after proving an optimal estimate for the moment of inertia for hypersurfaces, with geodesic hyperspheres as limitting hypersurfaces, we study the associated pinching problem (Theorem 6.3). It turns out that the stability of the above eigenvalues estimates is closely linked to the pinchng of the moment of inertia (Proposition 6.8).

\section{Preliminaries : Hypersurfaces of Weighted Euclidean sPaCe}

A weighted manifold $\left(\bar{M}, \bar{g}, d \bar{\mu}_{f}\right)$ is a Riemannian manifold $(\bar{M}, \bar{g})$ endowed with a weighted volume form $d \bar{\mu}_{f}=e^{-f} d v_{\bar{g}}$, where $f$ is a real-valued smooth function on $\bar{M}$ and $d v_{\bar{g}}$ is the Riemannian volume form associated with the metric $\bar{g}$. In the present paper, we will focus on the case where $(\bar{M}, \bar{g})$ is the Euclidean space $\left(\mathbb{R}^{N}\right.$, can $)$ with its canonical flat metric and we will consider isometric immersions of Riemannian manifolds $\left(M^{n}, g\right)$ into $\left(\mathbb{R}^{N}\right.$, can $)$. For such an immersion, we can define on $M$ a divergence associated with the volume form $d \mu_{f}=e^{-f} d v_{g}$ by

$$
\operatorname{div}_{f} Y=\operatorname{div} Y-\langle\nabla f, Y\rangle
$$

or equivalently

$$
d\left(\iota_{Y} d \mu_{f}\right)=\operatorname{div}_{f}(Y) d \mu_{f},
$$

where $\nabla$ is the gradient on $M$, that is the projection on $T M$ of the gradient $\bar{\nabla}$ on $\bar{M}$. We call it the $f$-divergence. We recall briefly by some basic facts about the 
$f$-divergence. We first have the weighted version of the divergence theorem:

$$
\int_{M} \operatorname{div}_{f} Y d \mu_{f}=0
$$

for any vector field $Y$ on $M$. From this, we deduce easily the integration by parts formula

$$
\int_{M} u \operatorname{div}_{f} Y d \mu_{f}=-\int_{M}\langle\nabla u, Y\rangle d \mu_{f},
$$

for any smooth function $u$ and any vector field $Y$ on $M$. From this we can prove the following weighted version of the so called Hsiung-Minkowski formula. For any divergence-free symmetric $(1,1)$-tensor, we have

$$
\int_{M}\left(\left\langle X, H_{T}-T \nabla f\right\rangle+\operatorname{tr}(T)\right) d \mu_{f}=0,
$$

where $H_{T}=\sum_{i, j=1}^{n}\left\langle T e_{i}, e_{j}\right\rangle B\left(e_{i}, e_{j}\right)$, with $B$ the second fundamental form and $\left\{e_{1}, \cdots, e_{n}\right\}$ a local orthonormal frame of $T M$ (see 33. for the proof). Note that in the case without density, the classical Hsiung-Minkowski formula (see [22] for $T=\mathrm{Id})$ is given by

$$
\int_{M}\left(\left\langle X, H_{T}\right\rangle+\operatorname{tr}(T)\right) d v_{g}=0
$$

Finally, we recall that in this weighted context, Lichnerowicz [26] and Bakry-Émery [6] introduced the natural generalizations of the Ricci curvature

$$
\operatorname{Ric}_{f}=\operatorname{Ric}+\operatorname{Hess} f,
$$

which known as Bakry-Émery tensor or $\infty$-Bakry-Émery tensor as it appears as a special case of $N$-Bakry-Émery tensors defined by

$$
\operatorname{Ric}_{f}^{N}=\operatorname{Ric}_{f}-\frac{1}{N} d f \otimes d f,
$$

for $N>0$. This curvature tensor is the natural generalization of the Ricci curavture whic allows extensions of many classical comparison results in Ricci curvature bounded form below in the case of smooth metric measure spaces. One can refere for instance to [36] for a survey about these questions.

We finish this section of preliminaries by recalling a useful elementary lemma. Let $\left(M^{n}, g\right)$ be a connected and oriented closed Riemannian manifold isometrically immersed by $X$ into $\mathbb{R}^{N}$. We denote by $\left\{\partial_{1}, \cdots, \partial_{N}\right\}$ the canonical frame of $\mathbb{R}^{N}$ and for $k \in\{1, \cdots, N\}, X^{k}=\left\langle X, \partial_{k}\right\rangle$ the coordinate functions. Then, we have the following elementary lemma.

Lemma 2.1. If $A$ is a field of endomorphisms on $M$, we have

$$
\sum_{k=1}^{N}\left\langle A\left(\nabla X^{k}\right), \nabla X^{k}\right\rangle=\operatorname{tr}(A) .
$$

The proof can be found in 33 . 


\section{UPPER BOUNDS FOR STEKLOV-TYPE PROBLEMS}

3.1. Generalized Steklov problems. In 25, Ilias and Makhoul proved analogues of inequalities (1) and (2) for the first eigenvalue of the Steklov problem for submanifolds with boundary of the Euclidean space $\mathbb{R}^{N}$. Let a compact Riemannian manifold $(M, \bar{g})$ with non-empty boundary $\partial M$. The induced metric on $\partial M$ will be denoted $g$. We consider the boundary problem

$$
\begin{cases}\Delta u=0 & \text { on } M \\ \frac{\partial u}{\partial \nu}=\sigma u & \text { on } \partial M\end{cases}
$$

where $\sigma$ is a real number and $u$ a $\mathcal{C}^{2}$ function over $M$. This problem was introduces by Steklov [35] and has been widely studied (see [25] and reference therein). It is a well-known fact that the set of all $\sigma$ for which this problem admits solutions is an increasing sequence of nonnegative real numbers. This sequence is exactely the spectrum of the so-called Dirichlet-to-Neumann operator. In [25, Ilias and Makhoul proved the following Reilly-type inequality for the first eigenvalue of this Steklov spectrum

$$
\sigma_{1}\left(\int_{\partial M} H_{r} d v_{g}\right)^{2} \leqslant n \mathrm{~V}(M) \int_{\partial M}\left\|H_{r+1}\right\|^{2} d v_{g}
$$

which is also valid for any $r$ if $n=N-1$ and only for even $r$ if $N>n-1$. As for the Reilly inequalities (1) and (2), equality in (8) is characterized.

In this section, we will consider some generalized Steklov problems. Let $f$ be a smooth function on $M$, we can define on $M$ a Laplace operator associated with the volume form $\bar{\mu}_{f}=e^{-f} d v_{\bar{g}}$ by

$$
\bar{\Delta}_{f} u=-\operatorname{div}_{f}(\bar{\nabla} u)=\bar{\Delta} u+\langle\bar{\nabla} f, \bar{\nabla} u\rangle,
$$

where $\bar{\nabla}$ is the gradient on $M$. We call it the $f$-Laplacian which is often called Bakry-Émery Laplacian, Witten Laplacian or drifting Laplacian in the litterature. Moreover, for a positive symmetric divergence-free $(1,1)$-tensor $T$, we consider the operator $L_{T, f}$ by

$$
L_{T, f} u=-\operatorname{div}_{f}(T \bar{\nabla} u),
$$

for any $\mathcal{C}^{2}$ fonction $u$ on $M$. Then, we can consider the following generalized weighted Steklov problem

$$
\begin{cases}L_{T, f} u=0 & \text { on } M \\ \frac{\partial u}{\partial \nu_{T}}=\sigma u & \text { on } \partial M\end{cases}
$$

For $T=\mathrm{Id}$, this problem has be considered recently by Batista and Santos [9] in the instrinsic setting. Namely considering domains in a manifold of nonnegative Bakry-Émery Ricci curvature, they obtain lower bound for the eigenvalue of this weighted Steklov problem. Here, we will derive upper bounds for domains of a manifold lying in a Euclidean space.

Precisely, we consider the generalized Stelkov problem (9) with the presence of a 
tensor $T$. From [5], we know that this problem also have a discrete nonnegative spectrum and we denote by $\sigma_{1, T, f}$ its first eigenvalue.

In this first result, we obtain Reilly-type inequalities similar to those obtained in 33] for $\lambda_{1}\left(L_{T, f}\right)$ and generalizing the results of [25] when the ambiant space is endowed with a measure with density. Namely, we prove following

Theorem 3.1. Let $\left(M^{n}, g\right)$ be a connected and oriented closed Riemannian manifold isometrically immersed into the Euclidean space $\mathbb{R}^{N}$ endowed with a density $e^{-f}$. Let $T$ be a positive symmetric divergence-free $(1,1)$-tensor over $M$ and $S$ a symmetric divergence free $(1,1)$-tensor over $\partial M$. Then, the first eigenvalue of the generalized Steklov problem satisifes

$$
\sigma_{1, T, f}\left(\int_{\partial M} \operatorname{tr}(S) \mu_{f}\right)^{2} \leqslant\left(\int_{M} \operatorname{tr}(T) \bar{\mu}_{f}\right) \int_{\partial M}\left(\left\|H_{S}\right\|^{2}+\|S \nabla f\|^{2}\right) \mu_{f} .
$$

Moreover,

- If $f$ is constant, $H_{S}$ does not vanish identically and if equality occurs, then $\operatorname{tr}(S)$ and $\left\|H_{S}\right\|$ are non-zero constants, $M$ is T-minmally immersed into $\mathbb{R}^{N}$ and $\partial M$ lies into a geodesic hypersphere of $\mathbb{R}^{N}$ of radius $\frac{|\operatorname{tr}(S)|}{\| H_{S}||}$.

- If $f$ is not constant and if equality holds in the case $S=\operatorname{Id}$ then $M$ is a self-shrinker for the mean curvature flow and $f_{\mid M}=a-\frac{c}{2} r_{p}^{2}$, where $r_{p}$ is the Euclidean distance to the center of mass $p$ of $M$. In particular, if $n=N-1$ and $H>0$ or $n=2, N=3$ and $M$ is embedded and has genus 0 , then $M$ a geodesic hypersphere.

Remark 3.2. Note that $\bar{\mu}_{f}$ and $\mu_{f}$ denote respectively the weighted measure on $M$ and $\partial M$.

Proof: Up to a translation, we can assume that for coordinates functions $X^{k}$ satisfy $\int_{M} X^{k} d v_{g}=0$. Hence, from the variational characterization of $\sigma_{1, T, f}$, using the coordinates $X^{k}$ as test functions and taking the sum for $k$ from 1 to $N$ we have

$$
\sigma_{1, T, f} \sum_{k=1}^{N} \int_{\partial M}\left|X^{k}\right|^{2} \mu_{f} \leqslant \sum_{k=1}^{N} \int_{M}\left\langle T \nabla X^{k}, X^{k}\right\rangle \bar{u}_{f},
$$

which gives, by Lemma 2.1

$$
\sigma_{1, T, f} \int_{\partial M}|X|^{2} \mu_{f} \leqslant \int_{M} \operatorname{tr}(T) \bar{\mu}_{f} .
$$

Now, we multiply this last inequality by $\int_{\partial M}\left(\left\|H_{S}\right\|^{2}+\|S \nabla f\|^{2}\right) \mu_{f}$ and we use the Hsiung-Minkowski formula (6) on $\partial M$ with the tensor $S$ to get

$$
\sigma_{1, T, f}\left(\int_{\partial M} \operatorname{tr}(S) \mu_{f}\right)^{2} \leqslant\left(\int_{M} \operatorname{tr}(T) \bar{\mu}_{f}\right) \int_{\partial M}\left(\left\|H_{S}\right\|^{2}+\|S \nabla f\|^{2}\right) \mu_{f} .
$$

The inequality is proven. Let us consider the equality case. First, assume that $f=0, H_{S}$ does not vanish identically and equality occurs. Since equality occurs, then, equality holds in the Cauchy-Schwarz inequality, that is, $H_{S}=\lambda X$ on $\partial M$ for some non-zero constant $\lambda$. This implies that, on $\partial M$, the position vector $X$ 
is normal. Clearly, we deduce that for any vector field $Y$ tangent to $\partial M$, we have $Y\left(\|X\|^{2}\right)=2\left\langle\bar{\nabla}_{Y} X, X\right\rangle=\langle Y, X\rangle=0$ and so $X$ has constant norm on $\partial M$. Therefore the same holds for $H_{S}$ since $H_{S}=\lambda X$.

Moreover, since, always on $\partial M, X^{T}=0$, we deduce that

$$
0=\operatorname{tr}(S)+\left\langle X, H_{S}\right\rangle .
$$

Indeed, we recall the following classical fact (see [33] for instance)

$$
\operatorname{div}\left(S X^{T}\right)=\operatorname{tr}(S)+\left\langle X, H_{S}\right\rangle
$$

which after intrgartion gives the Hsiung-Minkowski formula. Hence, we get

$$
\operatorname{tr}(S)=-\left\langle X, H_{S}\right\rangle=-\frac{1}{\lambda}\left\|H_{S}\right\| .
$$

This implies that $\operatorname{tr}(S)$ is also a non-zero constant and $\|X\|_{\partial M}=\frac{|\operatorname{tr}(S)|}{\left\|H_{S}\right\|}$, that is, $\partial M$ lies into a geodesic sphere of radius $\frac{|\operatorname{tr}(S)|}{\left\|H_{S}\right\|}$.

Now, we prove the last consequence of the equality case. Equality implies that the coordinate functions $X^{i}$ are eigenfunctions of the generalized Steklov problem. Hence, they satisfy $L_{T} X^{i}=0$. But, since, on $M, L_{T} X=-H_{T}$ (see 20] or 33] for instance), we get immediately that $H_{T}=0$, that is $M$ lies $T$-minimally into $\mathbb{R}^{N}$.

Now, assume that $f$ is not constant and $S=$ Id. If equality occurs then all the above inequalities are equalities. In particular, equality occurs in the CauchySchwarz inequality and we have $H-\nabla f=c X$ for some constant $c$. Identifying tangential and normal parts, we get $\nabla f=-c X^{\top}$ and $H=c X^{\perp}$.

The normal equation $H=c X^{\perp}$ is exactly the definition of a self-similar solution of the mean curvature flow. Since $M$ is a compact submanifold of $\mathbb{R}^{N}, c$ cannot be zero. The case $c>0$ is no more possible. Indeed, if $c>0$, then $M$ is a self-expander, but it is well known that there exists no compact self-expander. Hence, the only possibility is $c<0$, that is $M$ is a self-shrinker.

In addition, since $X^{\top}=\frac{1}{2} \nabla\|X\|^{2}$, the tangential equation becomes $\nabla\left(f+\frac{c}{2}\|X\|^{2}\right)=0$. Since $M$ is connected, there exists a constant a such that $f_{\mid M}=a-\frac{c}{2}\|X\|^{2}$.

In the particular cases $N=n+1$ and $H>0$ or $n=2, N=3$ and $M$ is embedded and has genus 0 , we know from 23 and 11 respectively that $M$ has to be a geodesic hypersphere. This finishes the proof of the equality case. This concludes the proof.

3.2. Steklov-Wentzell problem. Let $\left(M^{n}, \bar{g}\right)$ be a smooth submanifold of the Euclidean space $\mathbb{R}^{N}$ with non-empty boundary $\partial M$ and $b$ a positive constant. We will denote by $g$ the induced metric on $\partial M$ and the Laplacian on $M$ and $\partial M$ will be denoted respectively by $\bar{\Delta}$ and $\Delta$. We consider the following Steklov-type problem for the Laplacian $\bar{\Delta}$ with the so-called Wentzell boundary condition. Namely, we 
consider

$$
\begin{cases}\bar{\Delta} u=0 & \text { on } M \\ -b \Delta u-\frac{\partial u}{\partial \nu}=\alpha u & \text { on } \partial M\end{cases}
$$

Obviously, if $b=0$, then, we recover the classical Steklov problem. The spectrum of this problem is an increasing sequence (see [15])

$$
0=\alpha_{0}<\alpha_{1} \leqslant \alpha_{2} \cdots \leqslant \alpha_{k} \leqslant \cdots \longrightarrow+\infty
$$

The eigenvalue 0 is simple and the corresponding eigeinfunctions are the constant ones. Moreover (see [15, 37]), $\alpha_{1}$ has the following variational characterization

$$
\alpha_{1}=\inf \left\{\frac{\int_{M}\|\bar{\nabla} u\|^{2} d v_{\bar{g}}+b \int_{\partial M}\|\nabla u\|^{2} d v_{g}}{\int_{\partial M} u^{2} d v_{g}} \mid \int_{\partial M} u d v_{g}=0\right\} .
$$

We prove the following upper bound for the first positive eigenvalue $\alpha_{1}$ of this problem

Theorem 3.3. Let $\left(M^{n}, g\right)$ be a connected and oriented compact Riemannian manifold with non-empty boundary $\partial M$ isometrically immersed into the Euclidean space $\mathbb{R}^{N}$. Let $S$ be a symmetric divergence free $(1,1)$-tensor over $\partial M$. Then, the first eigenvalue of the Steklov-Wentzell problem satisifes

$$
\alpha_{1}\left(\int_{\partial M} \operatorname{tr}(S) d v_{g}\right)^{2} \leqslant(n V(M)+b(n-1) V(\partial M))\left(\int_{\partial M}\left\|H_{S}\right\|^{2} d v_{g}\right) .
$$

Moreover, if $H_{S}$ does not vanish identically, then if equality occurs then $\operatorname{tr}(S)$ and $\left\|H_{S}\right\|$ are non-zero constants, $M$ is a minmal submanifold of $\mathbb{R}^{N}$ and $\partial M$ lies into a geodesic hypersphere of $\mathbb{R}^{N}$ of radius $\frac{|\operatorname{tr}(S)|}{\left\|H_{S}\right\|}$.

In particular, if $n=N$, then equality occurs if and only if $M$ is a geodesic disk of radius $\frac{|\operatorname{tr}(S)|}{\left\|H_{S}\right\|}$.

Proof: Here again, we can assume that for any $k \in\{1, \cdots, N\}, \int_{M} X^{k} d v_{g}=0$ and so use the coordinates functions in 12 to get

$$
\alpha_{1} \int_{\partial M}\|X\|^{2} d v_{g} \leqslant \sum_{i=1}^{N}\left(\int_{M}\left\|\bar{\nabla} X^{i}\right\|^{2} d v_{\bar{g}}+b \int_{\partial M}\left\|\nabla X^{i}\right\|^{2} d v_{g}\right)
$$

First, by Lemma 2.1 applied respectively on $M$ and $\partial M$, we have $\sum_{i=1}^{N}\left\|\bar{\nabla} X^{i}\right\|^{2}=n$ and $\sum_{i=1}^{N}\left\|\nabla X^{i}\right\|^{2}=n-1$ and so we obtain from 13.

$$
\alpha_{1} \int_{\partial M}|X|^{2} d v_{g} \leqslant n V(M)+(n-1) V(\partial M) .
$$


Using (14), the Cauchy-Schwarz inequality and the Hsiung-Minkowski formula. We have

$$
\begin{aligned}
\alpha_{1}\left(\int_{\partial M} \operatorname{tr}(S) d v_{g}\right)^{2} & \leqslant \alpha_{1}\left(\int_{\partial M}\left\langle X, H_{S}\right\rangle d v_{g}\right)^{2} \\
& \leqslant \alpha_{1}\left(\int_{\partial M}\|X\|^{2} d v_{g}\right)\left(\int_{\partial M}\left\|H_{S}\right\|^{2} d v_{g}\right) \\
& \leqslant(n V(M)+(n-1) V(\partial M))\left(\int_{\partial M}\left\|H_{S}\right\|^{2} d v_{g}\right),
\end{aligned}
$$

which is the desired inequality.

Now, if $H_{S}$ does not vanish identically and equality occurs, then as in the proof of Theorem 3.1 , we get that $\left\|H_{S}\right\|$ and $\operatorname{tr}(S)$ are non-zero constants and $\partial M$ lies into a geodesic sphere of radius $\frac{|\operatorname{tr}(S)|}{\left\|H_{S}\right\|}$. Moreover, since equality occurs, the coordinates $\left\{X_{i}\right\}_{i=1, \cdots, N}$, used as test functions, satisfiy $\bar{\Delta} X^{i}=0$ over $M$ and so $M$ is immersed minimally into $\mathbb{R}^{N}$.

3.3. Biharmonic Steklov problem. Now, we consider the following biharmonic Steklov problem. Let $\left(M^{n}, \bar{g}\right)$ be a smooth submanifold of the Euclidean space $\mathbb{R}^{N}$ with non-empty boundary $\partial M$ and $\tau$ a positive constant.

$$
\begin{cases}\bar{\Delta}^{2} u-\tau \bar{\Delta} u=0 & \text { on } M \\ \frac{\partial^{2} u}{\partial \nu^{2}}=0 & \text { on } \partial M \\ \tau \frac{\partial u}{\partial \nu}-\operatorname{div}_{\partial M}\left(P_{\partial M}\left(\left(\nabla^{2} u\right) \nu\right)\right)-\frac{\partial \Delta u}{\partial \nu}=\beta u & \text { on } \partial M .\end{cases}
$$

This problem has a discret spectrum consisting in an increasing sequence (see [])

$$
0=\beta_{0}<\beta_{1} \leqslant \beta_{2} \cdots \leqslant \beta_{k} \leqslant \cdots \longrightarrow+\infty .
$$

The eigenvalue 0 is simple and the corresponding eigeinfunctions are the constant function. It is proven in [12 that the boundary condition in this problem are the natural one so that the weak formulation of this problem is:

$$
\int_{M}\left(\left\langle\bar{\nabla}^{2} u, \bar{\nabla}^{2} \phi\right\rangle+\tau\langle\bar{\nabla} u, \bar{\nabla} \phi\rangle\right) d v_{\bar{g}}=\beta \int_{\partial M} u \phi d v_{g}
$$

and where we have denoted by $g$ the induced metric on $\partial M$, Hence, the first positive eigenvalue $\beta_{1}$ has the following variational characterization

$$
\beta_{1}=\inf \left\{\frac{\int_{M}\left(\left\|\bar{\nabla}^{2} u\right\|^{2}+\tau\|\bar{\nabla} u\|^{2}\right) d v_{\bar{g}}}{\int_{\partial M} u^{2} d v_{g}} \mid \int_{\partial M} u d v_{g}=0\right\} .
$$

Theorem 3.4. Let $\left(M^{n}, g\right)$ be a connected and oriented compact Riemannian manifold with non-empty boundary $\partial M$ isometrically immersed into the Euclidean space $\mathbb{R}^{N}$. Let $S$ be a symmetric divergence free $(1,1)$-tensor over $\partial M$. Then, the first 
eigenvalue of the biharmonic Steklov problem satisifes

$$
\beta_{1}\left(\int_{\partial M} \operatorname{tr}(S) d v_{g}\right)^{2} \leqslant\left(\int_{M}\left(\|\bar{B}\|^{2}+n \tau\right) d v_{\bar{g}}\right)\left(\int_{\partial M}\left\|H_{S}\right\|^{2} d v_{g}\right)
$$

where $\bar{B}$ is the second fundamental form of $M$ in $\mathbb{R}^{N}$.

Moreover, if $H_{S}$ does not vanish identically, then if equality occurs then $\operatorname{tr}(S)$ and $\left\|H_{S}\right\|$ are non-zero constants, $M$ is a $\tau$-biharmonic submanifold of $\mathbb{R}^{N}$ and $\partial M$ lies into a geodesic hypersphere of $\mathbb{R}^{N}$ of radius $\frac{|\operatorname{tr}(S)|}{\left\|H_{S}\right\|}$.

In particular, if $n=N$, then

$$
\beta_{1}\left(\int_{\partial M} \operatorname{tr}(S) d v_{g}\right)^{2} \leqslant n \tau V(M)\left(\int_{\partial M}\left\|H_{S}\right\|^{2} d v_{g}\right)
$$

and equality occurs if and only if $M$ is a geodesic disk of radius $\frac{|\operatorname{tr}(S)|}{\left\|H_{S}\right\|}$.

Proof: We use coordinates as test functions (after a tranlsation if needed) in the variational characterization to get

$$
\beta_{1} \int_{\partial M}\|X\|^{2} d v_{g} \leqslant \sum_{i=1}^{N} \int_{M}\left(\left\|\bar{\nabla}^{2} X^{i}\right\|^{2}+\tau\left\|\bar{\nabla} X^{i}\right\|^{2}\right) d v_{\bar{g}}
$$

First, by Lemma 2.1, we have $\sum_{i=1}^{N} \tau\left\|\bar{\nabla} X^{i}\right\|^{2}=n \tau$. Moreover, we have the following elementary lemma

Lemma 3.5. We have

$$
\sum_{i=1}^{N}\left\|\bar{\nabla}^{2} X^{i}\right\|^{2}=\|\bar{B}\|^{2}
$$

where $\bar{B}$ is the second fundamental form of $M$ into $\mathbb{R}^{N}$.

Proof: Let $p \in M$ and take $\left\{e_{1}, \cdots, e_{n}\right\}$ a normal frame of $T_{p} M$. Then, we have

$$
\begin{aligned}
\left(\bar{\nabla}^{2} X^{i}\right)_{j k} & =e_{j}\left(e_{k}\left(X^{i}\right)\right) \\
& =e_{j}\left(\left\langle e_{k}, \partial_{i}\right\rangle\right) \\
& =\left\langle\nabla_{e_{j}}^{0} e_{k}, \partial_{i}\right\rangle
\end{aligned}
$$

where $\left\{\partial_{1}, \cdots, \partial_{N}\right\}$ is the canonical basis of $\mathbb{R}^{N}$ and $\nabla^{0}$ is the connection of the Euclidean metric of $\mathbb{R}^{N}$. Now, since $\left\{e_{1}, \cdots, e_{n}\right\}$ is a normal frame, we have $\nabla_{e_{j}} e_{k}=0$ and so $\nabla_{e_{j}}^{0} e_{k}=\bar{B}\left(e_{j}, e_{k}\right)$. Finally, $\left(\bar{\nabla}^{2} X^{i}\right)_{j k}=\left\langle\bar{B}\left(e_{j}, e_{k}\right), \partial_{i}\right\rangle$. Now, by summing 
over $i$ from 1 to $N$ we get

$$
\begin{aligned}
\sum_{i=1}^{N}\left\|\bar{\nabla}^{2} X^{i}\right\|^{2} & =\sum_{i=1}^{N} \sum_{j, k=1}^{n}\left(\left(\bar{\nabla}^{2} X^{i}\right)_{j k}\right)^{2} \\
& =\sum_{i=1}^{N} \sum_{j, k=1}^{n}\left\langle\bar{B}\left(e_{j}, e_{k}\right), \partial_{i}\right\rangle^{2} \\
& =\sum_{j, k=1}^{n}\left\|\bar{B}\left(e_{j}, e_{k}\right)\right\|^{2} \\
& =\|\bar{B}\|^{2}
\end{aligned}
$$

Now, putting $\sum_{i=1}^{N} \tau\left\|\nabla X^{i}\right\|^{2}=n \tau$ and Lemma 3.5 into [16], we get

$$
\beta_{1} \int_{\partial M}\|X\|^{2} d v_{g} \leqslant \int_{M}\left(\|\bar{B}\|^{2}+n \tau\right) d v_{\bar{g}}
$$

The proof of the inequality is a combination of (14), the Cauchy-Schwarz inequality and the Hsiung-Minkowski formula (7). We have

$$
\begin{aligned}
\beta_{1}\left(\int_{\partial M} \operatorname{tr}(S) d v_{g}\right)^{2} & \leqslant \beta_{1}\left(\int_{\partial M}\left\langle X, H_{S}\right\rangle d v_{g}\right)^{2} \\
& \leqslant \beta_{1}\left(\int_{\partial M}\|X\|^{2} d v_{g}\right)\left(\int_{\partial M}\left\|H_{S}\right\|^{2} d v_{g}\right) \\
& \leqslant\left(\int_{M}\left(\|\bar{B}\|^{2}+n \tau\right) d v_{\bar{g}}\right)\left(\int_{\partial M}\left\|H_{S}\right\|^{2} d v_{g}\right),
\end{aligned}
$$

which is the desired inequality.

Now, here again, if $H_{S}$ does not vanish identically and equality occurs, then as in the proof of Theorem 3.1. we get that $\left\|H_{S}\right\|$ and $\operatorname{tr}(S)$ are non-zero constants and $\partial M$ lies into a geodesic sphere of radius $\frac{|\operatorname{tr}(S)|}{\left\|H_{S}\right\|}$.

In addition, equality implies that the coordinate functions $X^{i}$ are eigenfunctions of the biharmonic Steklov problem. In particular, they satisfy $\bar{\Delta}^{2} X-\tau \bar{\Delta} X=0$ on $M$, that is, $M$ is a $\tau$-biharmonic submanifold of $\mathbb{R}^{N}$. This concludes the proof.

\section{UPPER BOUNDS FOR PANEITZ-LIKE OPERATORS}

On 4-dimensional Riemannian manifold $\left(M^{4}, g\right)$, the Paneitz operator, first introduced in [27] by Paneitz (see also [28, is the fourth order differential operator defined by

$$
P u=\Delta^{2} u-\operatorname{div}\left(\frac{2}{3} R \nabla u-2 \operatorname{Ric}(\nabla u)\right),
$$

for any smooth function $u$, where div is the divergence, $\Delta=-\operatorname{div} \nabla$ the Laplacian, $R$ the scalar curvature and Ric the $(1,1)$-Ricci tensor associated with the metric $g$. We will denote by ric the associated $(2,0)$-tensor. It has been generalized in any 
dimension by Branson [10]. Namely, we have for $n \geqslant 5$,

$$
P u=\Delta^{2} u--\operatorname{div}\left(\frac{(n-2)^{2}+4}{2(n-1)(n-2)} R \nabla u-\frac{4}{n-2} \operatorname{Ric}(\nabla u)\right)+\frac{n-4}{2} Q u,
$$

where $Q$ is the Branson $Q$-curvature associated with the metric $g$. The Paneitz operator is conformally covariant and plays a crucial role in the problem of prescribing $Q$-curvature. In the last two decades, the Paneitz operator (and its links with $Q$-curvature) has been intensively studied by many authors (see [16] for instance).

In the present section, we are not interested in the conformal aspect of the Paneitz operator but with its spectrum. In particular, we will give estimates of the first eigenvalue of the Paneitz operator, when the 4-dimensional manifold $\left(M^{4}, g\right)$ is a submanifold (especially a hypersurface) of a Euclidean space. In fact, we will consider the so-called Paneitz-like operators for which the classical Paneitz operator in dimension 4 is a particular case. We obtain general upper bounds which contain the estimates proved by Chen and $\mathrm{Li}$ in $[13$. The Paneitz-like operators are definied for some constant $a$ and $b$ by

$$
P_{a, b} u=\Delta^{2} u-\operatorname{div}(a R \nabla u+b \operatorname{Ric} \nabla u),
$$

for any smooth function $u$ on $M$. The positivity of $P_{a, b}$ is ensured if $n a+b \geqslant 0$ (see 38). Here, we consider the weighted version of these operators. Namely, we consider the following

$$
P_{f}^{a, b} u=\Delta_{f}^{2} u-\operatorname{div}_{f}\left(a R_{f} \nabla u+b \operatorname{Ric}_{f} \nabla u\right),
$$

where $\operatorname{div}_{f}, \Delta_{f}$ and $\operatorname{Ric}_{f}$ are respectively the $f$-divergence, the $f$-Laplacian and the Bakry-Émery Ricci curvature defined in Section 2. Moreover, we define $R_{f}$ as the trace of $\operatorname{Ric}_{f}$.

Theorem 4.1. Let $\left(M^{n}, g\right)$ be a closed Riemannian manifold isomtrically immersed into the weighted Euclidean space $\mathbb{R}^{N}$ endowed with the density $e^{-f}$. Let $T$ be a symmetric and divergence-free $(1,1)$-tensor on TM. Assume that the weighted Paneitzlike operator $P_{f}^{a, b}$ is positive. Then, the first non-zero eigenvalue $\Lambda_{1}$ of $P_{f}^{a, b}$ satisfies

$\Lambda_{1}\left(\int_{M} \operatorname{tr}(T) \mu_{f}\right)^{2} \leqslant\left(\int_{M}\left(n^{2}|H|^{2}+|\nabla f|^{2}+(n a+b) R_{f}\right) \mu_{f}\right) \int_{M}\left(\left|H_{T}\right|^{2}+\|T \nabla f\|^{2}\right) \mu_{f}$.

Moreover,

- If $f$ is constant, $H_{T}$ does not vanish identically and if equality occurs, then $\operatorname{tr}(T)$ and $\left|H_{T}\right|$ are non-zero constants and $M$ is immersed into a geodesic hypersphere of $\mathbb{R}^{N}$ of radius $\frac{|\operatorname{tr}(T)|}{\left|H_{T}\right|}$.

In particular, if $n=N-1, M$ is a geodesic hypersphere of radius $\frac{|\operatorname{tr}(T)|}{\left|H_{T}\right|}$.

- If $f$ is not constant and if equality holds in the case $S=\operatorname{Id}$ then $M$ is a self-shrinker for the mean curvature flow and $f_{\mid M}=a-\frac{c}{2} r_{p}^{2}$, where $r_{p}$ is the Euclidean distance to the center of mass $p$ of $M$. In particular, if $n=N-1$ and $H>0$ or $n=2, N=3$ and $M$ is embedded and has genus 0 , then $M$ a geodesic hypersphere.

Before giving the prove, we state this elementary lemma. 
Lemma 4.2. We have

$$
\Delta_{f} X=-n H+\nabla f
$$

Proof: The proof is elementary form the definition of $\Delta_{f}$. Indeed, we have

$$
\begin{aligned}
\Delta_{f} X & =\sum_{i=1}^{N} \Delta_{f} X^{i} \partial_{i} \\
& =\sum_{i=1}^{N} \Delta X^{i} \partial_{i}+\sum_{i=1}^{N}\left\langle\nabla X^{i}, \nabla f\right\rangle \partial_{i} \\
& =\Delta X+\sum_{i=1}^{N}\left\langle\partial_{i}, \nabla f\right\rangle \partial_{i} \\
& =-n H+\nabla f,
\end{aligned}
$$

where we have used the classical facts that $\Delta X=-n H$ and $\nabla X^{i}=\partial_{i}^{\top}$.

Proof of Theorem 4.1 First, if needed, we make a translation in order to assume that the center of mass of $X$ for the measure $\mu_{f}$ is 0 . Hence, we can use the coordinates $X^{1}, \cdots, X^{N}$ as test functions in the Rayleigh quotient to get

$$
\Lambda_{1} \int_{M}|X|^{2} \mu_{f} \leqslant \int_{M}\left\langle X, P_{f}^{a, b} X\right\rangle \mu_{f} .
$$

Now, we compute the term $\int_{M}\left\langle X, P_{f}^{a, b} X\right\rangle \mu_{f}$. We have

$$
\begin{aligned}
\int_{M}\left\langle X, P_{f}^{a, b} X\right\rangle \mu_{f} & =\sum_{k=1}^{N} \int_{M} X^{k} P_{f}^{a, b} X^{k} \mu_{f} \\
& =\sum_{k=1}^{N} \int_{M} X^{k} \Delta_{f}^{2} X^{k} \mu_{f}-\int_{M} \sum_{k=1}^{N} X^{k} \operatorname{div}_{f}\left(a R_{f} \nabla X^{k}+b \operatorname{Ric}_{f}\left(\nabla X^{k}\right)\right) \mu_{f} \\
& =\sum_{k=1}^{N} \int_{M}\left|\Delta_{f} X^{k}\right|^{2} \mu_{f}+\int_{M} \sum_{k=1}^{N}\left(a R_{f}\left|\nabla X^{k}\right|^{2}+b\left\langle\operatorname{Ric}_{f}\left(\nabla X^{k}\right), \nabla X^{k}\right\rangle\right) \mu_{f},
\end{aligned}
$$

where we have integrated by parts for the last line. Moreover, from Lemma 2.1. we have

$$
\sum_{k=1}^{N}\left|\nabla X^{k}\right|^{2}=n \quad \text { and } \quad \sum_{k=1}^{N} \operatorname{Ric}_{f}\left(\nabla X^{k},\left.\nabla X^{k}\right|^{2}\right)=\operatorname{tr}\left(\operatorname{Ric}_{f}\right)=R_{f} .
$$

By Lemma 4.2, we have $\Delta_{f} X=-n H+\nabla f$, which gives

$$
\int_{M}\left\langle X, P_{f}^{a, b} X\right\rangle \mu_{f}=\int_{M}\left(n^{2}|H|^{2}+|\nabla f|^{2}+(n a+b) R_{f}\right) \mu_{f}
$$

and therefore

$$
\Lambda_{1} \int_{M}|X|^{2} \mu_{f} \leqslant \int_{M}\left(n^{2}|H|^{2}+|\nabla f|^{2}+(n a+b) R_{f}\right) \mu_{f} .
$$


Now, we multiply by $\left(\int_{M} \operatorname{tr}(T) \mu_{f}\right)^{2}$ and we get

$$
\begin{aligned}
\Lambda_{1}\left(\int_{M} \operatorname{tr}(T) \mu_{f}\right)^{2} \int_{M}|X|^{2} \mu_{f} & \leqslant\left(\int_{M}\left(n^{2}|H|^{2}+|\nabla f|^{2}+(n a+b) R_{f}\right) \mu_{f}\right)\left(\int_{M} \operatorname{tr}(T) \mu_{f}\right)^{2} \\
& \leqslant\left(\int_{M}\left(\left.n^{2}|| H\right|^{2}+|\nabla f|^{2}+(n a+b) R_{f}\right) \mu_{f}\right)\left(\int_{M}\left\langle H_{T}-T \nabla f, X\right\rangle \mu_{f}\right)^{2}
\end{aligned}
$$

where we have used the generalized Hsiung-Minkowski formula (6). Then, using the Cauchy-Schwarz inequality for the pointwise scalar product, then the CauchySchwarz inequality for the $L^{2}$-scalar product and simplifying by $\int_{M}|X|^{2} \mu_{f}$ we get

$\Lambda_{1}\left(\int_{M} \operatorname{tr}(T) \mu_{f}\right)^{2} \leqslant\left(\int_{M}\left(n^{2}|H|^{2}+|\nabla f|^{2}+(n a+b) R_{f}\right) \mu_{f}\right)\left(\int_{M}\left|H_{T}-T \nabla f\right|^{2} \mu_{f}\right)$,

Finally, since $H_{T}$ and $T \nabla f$ are orthogonal, we get the wanted inequality

$\Lambda_{1}\left(\int_{M} \operatorname{tr}(T) \mu_{f}\right)^{2} \leqslant\left(\int_{M}\left(n^{2}|H|^{2}+|\nabla f|^{2}+(n a+b) \int_{M} R_{f}\right) \mu_{f}\right) \int_{M}\left(\left|H_{T}\right|^{2}+|T \nabla f|^{2}\right) \mu_{f}$,

where we have used that $\left|H_{T}-T \nabla f\right|^{2}=\left|H_{T}\right|^{2}+|T \nabla f|^{2}$ since $H_{T}$ is normal and $T \nabla f$ is tangent.

Now, assume that $H_{T}$ does not vanish identically. If equality occurs, then all the above inequalities become equalities. In particular, we have $H_{T}=c X$ from the equality case of Cauchy-Schwarz inequality, where $c$ is a non-zero constant. This means that the position vector $X$ is everywhere normal to $M$. But, on the other hand, since $\nabla|X|^{2}=X^{\top}$, we get that $\nabla|X|^{2}=0$. Hence, $|X|=r$ is constant and $M$ lies in a geodesic hypersphere of radius $r$. Moreover, since $H_{T}=c X$, we get that $\left|H_{T}\right|$ is also constant and from Equation (11), we conclude that $\operatorname{tr}(T)=$ $-\left\langle X, H_{T}\right\rangle=-\frac{1}{c}\left|H_{T}\right|^{2}$. Thus, $\operatorname{tr}(T)$ is also constant. Note that, since we assume that $H_{T}$ does not vanish identically, $\operatorname{tr}(T)$ and $\left|H_{T}\right|$ are non-zero constants and we have $r=\frac{|\operatorname{tr}(T)|}{\left|H_{T}\right|}$.

Now, we will show that the immersion of $M$ in this hypersphere $\mathbb{S}^{N-1}(r)$ is $T$ minimal, that is, $\widetilde{H}_{T}=0$, where is defined by

$$
\widetilde{H}_{T}=\sum_{i, j=1}^{n} T\left(e_{i}, e_{j}\right) \widetilde{B}\left(e_{i}, e_{j}\right),
$$

with $\widetilde{B}$ the second fundamental form of $M$ in $\mathbb{S}^{N-1}(r)$. It is well known that $B=\widetilde{B}+\bar{B}$ where $\bar{B}$ is the second fundamental form of $\mathbb{S}^{N-1}$ into $\mathbb{R}^{N}$ and is given by $\bar{B}_{i j}=-\frac{1}{r^{2}} \delta_{i j} X$. From this and the definition of $H_{T}$ and $\widetilde{H}_{T}$, we get

$$
\begin{aligned}
H_{T} & =\widetilde{H}_{T}-\frac{1}{r^{2}} \sum_{i, j}^{n} T\left(e_{i}, e_{j}\right) \delta_{i j} X \\
& =\widetilde{H}_{T}-\frac{1}{r^{2}} \operatorname{tr}(T) X \\
& =\widetilde{H}_{T}-\frac{\left|H_{T}\right|^{2}}{\operatorname{tr}(T)} X \\
& =\widetilde{H}_{T}+c X=\widetilde{H}_{T}+H_{T} .
\end{aligned}
$$

We deduce that $\widetilde{H}_{T}=0$. This concludes the proof. 
We deduce immediately the following corollary when $T$ is a tensor associated with a higher order mean curvature.

Corollary 4.3. Let $\left(M^{n}, g\right)$ be a closed Riemannian manifold isomtrically immersed into the Euclidean space $\mathbb{R}^{N}$. Assume that the Paneitz-like operator $P_{a, b}$ is positive.

(1) If $n \leqslant N-2$ and $k \in\{0, \cdots, n-1\}$ is an even integer, then, the first non-zero eigenvalue $\Lambda_{1}$ of $P_{a, b}$ satisfies

$\Lambda_{1}\left(\int_{M} H_{k} d v_{g}\right)^{2} \leqslant\left(\int_{M}\left(n^{2}|H|^{2}+(n a+b) \int_{M} R\right) d v_{g}\right) \int_{M}\left|H_{k+1}\right|^{2} d v_{g}$.

Morever, if $H_{k+1}$ does not vanish identically and if equality occurs, then $H_{k}$ and $\left|H_{k+1}\right|$ are non-zero constants and $M$ is $(k+1)$-minimally immersed into a geodesic hypersphere of $\mathbb{R}^{N}$ of radius $\frac{\left|H_{k}\right|}{\left|H_{k+1}\right|}$.

(2) If $n=N-1$, for any $k \in\{0, \cdots, n-1\}$, the first non-zero eigenvalue $\Lambda_{1}$ of $P_{a, b}$ satisfies

$$
\Lambda_{1}\left(\int_{M} H_{k} d v_{g}\right)^{2} \leqslant\left(\int_{M}\left(n^{2}|H|^{2}+(n a+b) \int_{M} R\right) d v_{g}\right) \int_{M} H_{k+1}^{2} d v_{g},
$$

Morever, if $H_{k+1}$ does not vanish identically and if equality occurs, then $H_{k}$ and $H_{k+1}$ are non-zero constants and $M$ is a geodesic hypersphere of $\mathbb{R}^{N}$ of radius $\frac{\left|H_{k}\right|}{\left|H_{k+1}\right|}$.

We finish this section by a result for the first eigenvalue of the Paneitz operator in dimension at least 5 . The proof is completely analogue to the previous one with $a=, b=$ and the presence of the order zero term $Q u$ in the definition of $P$. We state the following result without proof.

Theorem 4.4. Let $\left(M^{n}, g\right), n>4$, be a closed Riemannian manifold isomtrically immersed into the Euclidean space $\mathbb{R}^{N}$. Let $T$ be a symmetric and divergence-free $(1,1)$-tensor on TM. Assume that the Paneitz operator $P$ is positive. Then, the first non-zero eigenvalue $\Lambda_{1}$ of $P$ satisfies

$$
\begin{aligned}
\Lambda_{1}\left(\int_{M} \operatorname{tr}(T) d v_{g}\right)^{2} \leqslant & \left(\int_{M}\left(n^{2}|H|^{2}+\frac{n^{3}-4 n^{2}+12 n-4}{2(n-1)(n-2)} R\right) d v_{g}\right) \int_{M}\left|H_{T}\right|^{2} d v_{g} \\
& +\frac{n-4}{2}\|Q\|_{\infty}\left(\int_{M} \operatorname{tr}(T) d v_{g}\right)^{2} .
\end{aligned}
$$

Moreover, if $H_{T}$ does not vanish identically and equality occurs, then $\operatorname{tr}(T)$ and $\left\|H_{T}\right\|$ are non-zero constants $M$ is T-minimally immersed into a geodesic sphere of $\mathbb{R}^{N}$ of radius $\frac{|\operatorname{tr}(T)|}{\left\|H_{T}\right\|}$.

\section{Further inequalities}

In this section, we derive from the estimates of Sections 3 and 4 some other estimates. Namely, we can obtained estimates for submanifolds of spheres or projectives space by the mean of the classical immersions of these spaces in euclidean spaces 
(see Section 5.1 and 5.2 . We can obtain in Section 5.3 some isoperimetric estimates for embedded hypersurfaces

\subsection{Estimates in spheres.}

Corollary 5.1. First, we have the following corollaries for submanifolds of spheres. Let $\left(M^{n}, g\right)$ be a connected and oriented closed Riemannian manifold isometrically immersed into the Euclidean space $\mathbb{S}^{N}$ endowed with a density $e^{-f}$. Let $T$ be a positive symmetric divergence-free $(1,1)$-tensor over $M$ and $S$ a symmetric divergence free $(1,1)$-tensor over $\partial M$

$$
\sigma_{1, T, f}\left(\int_{\partial M} \operatorname{tr}(S) \mu_{f}\right)^{2} \leqslant\left(\int_{M} \operatorname{tr}(T) \bar{\mu}_{f}\right) \int_{\partial M}\left(\left\|H_{S}\right\|^{2}+\operatorname{tr}(S)^{2}+\|S \nabla f\|^{2}\right) \mu_{f} .
$$

Proof: The proof comes easily from Theorem 3.1. We denote by $\Phi$ the immersion of $M$ into $\mathbb{S}^{N}$ and $\phi$ its restriction to $\partial M$. We also consider the canonical immersion $i$ of $\mathbb{S}^{N}$ into $\mathbb{R}^{N+1}$ and we extend the weight $f$ defined on $\mathbb{S}^{N}$ to a weight $\tilde{f}$ on $\mathbb{R}^{N+1}$, for instance by taking $\widetilde{f}(x)=|x| f\left(\frac{x}{|x|}\right)$ for any $x \in \mathbb{S}^{N}$ and $\widetilde{f}(0)=0$. From Theorem 3.1 we have

$$
\left.\sigma_{1, T, f}\right)\left(\int_{\partial M} \operatorname{tr}(S) \mu_{f}\right)^{2} \leqslant\left(\int_{M} \operatorname{tr}(T) \bar{\mu}_{f}\right) \int_{\partial M}\left(\left|H_{S}^{\prime}\right|^{2}+|S \nabla \widetilde{f}|^{2}\right) \mu_{f},
$$

where $H_{S}^{\prime}$ is defined by $H_{S}=\sum_{i, j=1}^{n} S\left(e_{i}, e_{j}\right) B^{\prime}\left(e_{i}, e_{j}\right)$ with $B^{\prime}$ the second fundamental form of the immersion of $\partial M$ into $\mathbb{R}^{N+1}$. Obviously, the second fundamental forms $B$ of $\phi$ and $B^{\prime}$ of $i \circ \phi$ are linked by the relation $B^{\prime}=B-g \phi$. Hence, we get immediately $H_{S}^{\prime}=H_{S}-\operatorname{tr}(S) \phi$. Therefore, we deduce that $\left\|H_{S}^{\prime}\right\|^{2}=\left\|H_{S}\right\|^{2}+\operatorname{tr}(S)^{2}$, since $H_{S}$ and $\phi$ are orthogonal and $\|\phi\|=1$ since $M$ (and so $\partial M$ ) is contained in the sphere $\mathbb{S}^{N}$. Reporting this in $\sqrt{19}$, and since $f$ coincides with $\widetilde{f}$ on $M$, we have $\nabla \tilde{f}=\nabla f$ and so

$$
\sigma_{1, T, f}\left(\int_{\partial M} \operatorname{tr}(S) \mu_{f}\right)^{2} \leqslant\left(\int_{M} \operatorname{tr}(T) \bar{\mu}_{f}\right) \int_{\partial M}\left(\left\|H_{S}\right\|^{2}+\operatorname{tr}(S)^{2}+\|S \nabla f\|^{2}\right) \mu_{f} .
$$

This concludes the proof.

Corollary 5.2. Let $\left(M^{n}, g\right)$ be a connected and oriented compact Riemannian manifold with non-empty boundary $\partial M$ isometrically immersed into the sphere $\mathbb{S}^{N}$. Let $S$ be a symmetric divergence free $(1,1)$-tensor over $\partial M$. Then, the first eigenvalue of the biharmonic Steklov problem satisifes

$$
\beta_{1}\left(\int_{\partial M} \operatorname{tr}(S) d v_{g}\right)^{2} \leqslant\left(\int_{M}\left(\|\bar{B}\|^{2}+n+n \tau\right) d v_{\bar{g}}\right)\left(\int_{\partial M}\left\|H_{S}\right\|^{2}+\operatorname{tr}(S) d v_{g}\right) .
$$

5.2. Estimates in projective spaces. As mentionned in [25, in the case where $S=\mathrm{Id}$, we can obtain estimates for submanifolds of projective spaces. Indeed, proceeding as in the proof of Corollary 5.1 with the standard embeddings of the projective space $\mathbb{K} P^{n}, \mathbb{K}=\mathbb{R}, \mathbb{C}$ or $\mathbb{H}$, we get form Theorem 3.1

$$
\sigma_{1, T}\left(\mathrm{~V}_{f}(\partial M)\right)^{2} \leqslant\left(\int_{M} \operatorname{tr}(T) \bar{\mu}_{f}\right) \int_{\partial M}\left(\left\|H^{\prime}\right\|^{2}+\|\nabla f\|^{2}\right) \mu_{f}
$$


where $H^{\prime}$ is the mean curvature of $\partial M \hookrightarrow \mathbb{K} P^{n} \hookrightarrow \mathbb{R}^{N}$ as a submanifold of $\mathbb{R}^{N}$. The mean curvature of $\partial M$ in $\mathbb{K} P^{n}$ is denoted $H$. Straightforward computations show that $\left\|H^{\prime}\right\|^{2}=\|H\|^{2}+c(m)$, with

$$
c(m)= \begin{cases}\frac{2(m+1)}{2(m+2)} & \text { if } \mathbb{K}=\mathbb{R} \\ \frac{2(m}{m} & \text { if } \mathbb{K}=\mathbb{C} \\ \frac{2(m+4)}{m} & \text { if } \mathbb{K}=\mathbb{H},\end{cases}
$$

and where $m$ is the dimension of $M$ (see [19] for details). Note that $V_{f}$ denote the $f$-volume, that is, the volume for the measure $\mu_{f}$. Hence, we have

Corollary 5.3. Let $\left(M^{n}, g\right)$ be a connected and oriented closed Riemannian manifold with non-empty boundary $\partial M$, isometrically immersed into $\mathbb{M}^{N}$ endowed with a density $e^{-f}$. Let $T$ be a positive symmetric divergence-free $(1,1)$-tensor over $M$. Then, the first eigenvalue of the generalized Steklov problem associated to the operator $L_{T, f}$ satisfies

$$
\sigma_{1, T, f}\left(\mathrm{~V}_{f}(\partial M)\right)^{2} \leqslant\left(\int_{M} \operatorname{tr}(T) \bar{\mu}_{f}\right) \int_{\partial M}\left(\|H\|^{2}+\|\nabla f\|^{2}+c(m)\right) \mu_{f} .
$$

where $\mu$ is the measure on $M$ defined by $\mu_{f}=e^{-f} d v_{g}$ and with

$$
c(n)= \begin{cases}1 & \text { if } \mathbb{M}^{N}=\mathbb{S}^{N} \\ \frac{2(n+1)}{n} & \text { if } \mathbb{M}^{N}=\mathbb{R} P^{N} \\ \frac{2(n+2)}{n} & \text { if } \mathbb{M}^{N}=\mathbb{C} P^{N} \\ \frac{2(n+4)}{n} & \text { if } \mathbb{M}^{N}=\mathbb{H} P^{N} .\end{cases}
$$

This remark also holds for the estimates of Section 4. Indeed, we deduce immediately from Theorem 3.1 for $S=$ Id the following estimates.

Corollary 5.4. Let $\left(M^{n}, g\right)$ be a connected and oriented closed Riemannian manifold isometrically immersed into $\mathbb{M}^{N}$ endowed with a density $e^{-f}$. Let $T$ be a positive symmetric divergence-free $(1,1)$-tensor over $M$. Then, the first eigenvalue of the operator $L_{T, f}$ satisfies

$$
\lambda_{1}\left(L_{T, f}\right)\left(\mathrm{V}_{f}(M)\right)^{2} \leqslant\left(\int_{M} \operatorname{tr}(T) \mu\right)\left(\int_{M}\left(\|H\|^{2}+\|\nabla f\|^{2}+c(n)\right) \mu_{f}\right),
$$

where $\mu$ is the measure on $M$ defined by $\mu_{f}=e^{-f} d v_{g}$ and with

$$
c(n)= \begin{cases}1 & \text { if } \mathbb{M}^{N}=\mathbb{S}^{N} \\ \frac{2(n+1)}{n} & \text { if } \mathbb{M}^{N}=\mathbb{R} P^{N} \\ \frac{2(n+2)}{n} & \text { if } \mathbb{M}^{N}=\mathbb{C} P^{N} \\ \frac{2(n+4)}{n} & \text { if } \mathbb{M}^{N}=\mathbb{H} P^{N} .\end{cases}
$$

For Paneitz-like operators, we have the following Corollary obtained from Theorem 4.1 .

Corollary 5.5. Let $\left(M^{n}, g\right)$ be a connected and oriented closed Riemannian manifold with non-empty boundary $\partial M$, isometrically immersed into $\mathbb{M}^{N}$ endowed with a density $e^{-f}$. Assume that the weighted Paneitz-like operator $P_{f}^{a, b}$ is positive. Then, the first non-zero eigenvalue $\Lambda_{1}$ of $P_{f}^{a, b}$ satisfies

$\Lambda_{1} \mathrm{~V}_{f}(M) \leqslant\left(\int_{M}\left(n^{2}|H|^{2}+|\nabla f|^{2}+(n a+b) R_{f}+c(n)\right) \mu_{f}\right)\left(\int_{M}\left(\|H\|^{2}+\|\nabla f\|^{2}+c(n)\right) \mu_{f}\right)$, 
where $\mu$ is the measure on $M$ defined by $\mu_{f}=e^{-f} d v_{g}$ and with

$$
c(n)= \begin{cases}1 & \text { if } \mathbb{M}^{N}=\mathbb{S}^{N} \\ \frac{2(n+1)}{2(n+2)} & \text { if } \mathbb{M}^{N}=\mathbb{R} P^{N} \\ \frac{2(n+2)}{2(n+4)} & \text { if } \mathbb{M}^{N}=\mathbb{C} P^{N} \\ \text { if } \mathbb{M}^{N}=\mathbb{H} P^{N}\end{cases}
$$

5.3. Isoperimetric estimates for embedded hypersurfaces. We consider now the case where the hypersurface $M$ is embedded into $\mathbb{R}^{n+1}$ and so bounds a domain $\Omega$. In this case, we can derive easily some estimates for the three eigenvalue problems considered above in terms both volumes $V(M)$ and $V(\Omega)$.

Proposition 5.6. Let $\left(M^{n}, g\right)$ be a closed, connected and oriented manifold isometrically embedded into the Euclidean space $\mathbb{R}^{n+1}$ (hence bounding a simply connected domain $\Omega$ ). Let $T$ a symmetric $(1,1)$-tensor with vanishing divergence. Assume moreover that $T$ is positive definite. Then,

(1) the first eigenvalue of the generalized Steklov problem satisifes

$$
\sigma_{1, T} \leqslant \frac{V(M)}{(n+1)^{2} V(\Omega)^{2}}\left(\int_{\Omega} \operatorname{tr}(T) d v_{\bar{g}}\right) .
$$

(2) the first eigenvalue of the biharmonic Steklov problem satisifes

$$
\alpha_{1} \leqslant \frac{V(M)}{(n+1)^{2} V(\Omega)^{2}}((n+1) V(\Omega)+b n V(M)) .
$$

(3) the first eigenvalue of the Steklov-Wentzell problem satisifes

$$
\beta_{1} \leqslant \frac{\tau V(M)}{(n+1) V(\Omega)}
$$

(4) if the Paneitz-like operator $P^{a, b}$ is positive, then, the first non-zero eigenvalue $\Lambda_{1}$ of $P^{a, b}$ satisfies

$$
\Lambda_{1} \leqslant \frac{V(M)}{(n+1)^{2} V(\Omega)^{2}}\left(\int_{M}\left(n^{2}|H|^{2}+(n a+b) R\right) d v_{g}\right) .
$$

(5) the first eigenvalue of the operator $L_{T}$ satisfies

$$
\lambda_{1}\left(L_{T}\right) \leqslant \frac{V(M)}{(n+1)^{2} V(\Omega)^{2}} \int_{M} \operatorname{tr}(T) d v_{g} .
$$

Moreover, for each inequality, if equality occurs, then $M$ is a geodesic hypersphere (and $\Omega$ is a round ball).

Proof: The proof of these inequalities is based on the following elementary fact that on $\Omega$,

$$
\operatorname{div}(X)=n+1
$$


From this, we have

$$
\begin{aligned}
(n+1) V(\Omega) & =\int_{\Omega} \operatorname{div}(X) d v_{\bar{g}} \\
& =\int_{M}\langle X, \nu\rangle d v_{g} \\
& \leqslant \int_{M}|X| d v_{g},
\end{aligned}
$$

where we have used the Stokes formula. Now, by the Cauchy-Schwarz inequality, we get

$$
(n+1)^{2} V(\Omega)^{2} \leqslant \int_{M}|X|^{2} d v_{g}
$$

Now, using 20 we get immediately the five inequalities of Proposition 5.6 respectively with, 10, (14), 17), 18) and the well-known analogue for the operator $L_{T}$ (see [33])

$$
\lambda_{1}\left(L_{T}\right) \int_{M}|X|^{2} d v_{g} \leqslant \int_{M} \operatorname{tr}(T) d v_{g}
$$

\section{Pinching Results}

The common point is all the above inequalities is that we use coordinates as test functions together with an appropriate Hsiung-Minkowksi formula. Moreover for the codimension 1 case, equality occurs if and only if the hypersurfaces is a round sphere and it turns out that the moment of inertia $\|X\|_{2}$ of $M$ satisfies the limitting case of the following inequality.

Proposition 6.1. Let $\left(M^{n}, g\right)$ be a closed, connected and Riemannian manifold isomtrically immersed into Euclidean space $\mathbb{R}^{n+1}$ by $X$. Let $S$ be a symmetric and divergence-free $(1,1)$-tensor on $T M$. Then, we have

$$
\|X-\bar{X}\|_{2} \geqslant \frac{\left|\int_{M} \operatorname{tr}(S) d v_{g}\right|}{\left\|H_{S}\right\|_{2} V(M)} .
$$

Moreover, if $H_{S}$ does not vanish identically, then equality occurs if and only $\operatorname{tr}(S)$ and $\left\|H_{S}\right\|$ are non-zero constants and $X(M)$ is a geodesic sphere of radius $\frac{|\operatorname{tr}(S)|}{\left\|H_{S}\right\|}$.

Proof: For simplicity and without lost of generality, we assume that $\bar{X}=0$. The generalized Hsiung-Minkowksi formula says

$$
\int_{M}\left(\operatorname{tr}(S)+\left\langle H_{S}, X\right\rangle\right) d v_{g}
$$


Hence, we obtain by using the pointwise and then integral Cauchy-Schwarz inequality

$$
\begin{aligned}
\left|\int_{M} \operatorname{tr}(S) d v_{g}\right| & =\left|\int_{M}\left\langle H_{S}, X\right\rangle d v_{g}\right| \\
& \leqslant \int_{M}\left\|H_{S}\right\| \cdot|\langle X, \nu\rangle| d v_{g} \\
& \leqslant\left(\int_{M}\left\|H_{S}\right\|^{2} d v_{g}\right)^{1 / 2}\left(\int_{M}\langle X, \nu\rangle^{2} d v_{g}\right)^{1 / 2} \\
& \leqslant\left(\int_{M}\left\|H_{S}\right\|^{2} d v_{g}\right)^{1 / 2}\left(\int_{M}\|X\|^{2} d v_{g}\right)^{1 / 2} \\
& =\left\|H_{S}\right\|_{2}\|X\|_{2} V(M),
\end{aligned}
$$

which is the desired inequality.

Now, assume that $H_{S}$ does not vanish identically. If equality occurs, since $\left\|H_{S}\right\|_{2} \neq 0$, then equality occurs in the pointwise Cauchy-Schwarz inequality $|\langle X, \nu\rangle| \leqslant\|X\|$ at any point of $M$. This implies, as mentionned previously, that $X(M)$ is a geodesic sphere. We denote by $R$ the radius of this sphere. Now, equlity also imply equality in the integral Cauhcy -Schwarz inequality so $\left\|H_{S}\right\|$ and $|\langle X, \nu\rangle|=R$ are proportional and so $\left\|H_{S}\right\|$ is constant. Finally, since $X(M)$ is a geodesic sphere of radius $R$, its second fundamental form is $B=\frac{1}{R} g$. Hence, by definition of $H_{S}=\operatorname{tr}(B \circ S)$, get that $\left\|H_{S}\right\|=\frac{1}{R}|\operatorname{tr}(S)|$. This mean that $\operatorname{tr}(S)$ is also a non-zero constant and $R=\frac{|\operatorname{tr}(S)|}{\left\|H_{S}\right\|_{2}}$.

The converse is obvious.

As an immediate corollary, we get lower bounds for the extrinsic radius (see [30, 31] to compare with the known bounds in terms of mean curvatures).

Corollary 6.2. Let $\left(M^{n}, g\right)$ be a closed, connected and Riemannian manifold isomtrically immersed into Euclidean space $\mathbb{R}^{n+1}$ by $X$. Let $S$ be a symmetric and divergence-free $(1,1)$-tensor on $T M$. Then, the extrinsic radius $R(M)$ of $M$ satisfies

$$
R(M) \geqslant \frac{\left|\int_{M} \operatorname{tr}(S) d v_{g}\right|}{\left\|H_{S}\right\|_{2} V(M)} .
$$

Moreover, if $H_{S}$ does not vanish identically, then equality occurs if and only $\operatorname{tr}(S)$ and $\left\|H_{S}\right\|$ are non-zero constants and $X(M)$ is a geodesic sphere of radius $R(M)$ and $R(M)=\frac{|\operatorname{tr}(S)|}{\left\|H_{S}\right\|}$.

The inequality 21) implies as corollaries the lower bound of the extrinsic radius (Corollary 6.2 as well as the upper bounds for the operators $L_{T}$ (33]), Paneitzlike (Theorems 4.1) and for the generalized Steklov problem (Theorem 3.1), all for the non-weighted case and for hypersurfaces. Then, it is natural to think that a pinching result associated with (21) or another appropriate estimate of $\|X\|_{2}$ will implies pinchin results for the other inequalities cited above. And this is indeed the case as we will see in the sequel. 
We introduce the following pinching of the moment of inertia

$\left(I_{p, \varepsilon}\right)$

$$
\|X\|_{2} \leqslant \frac{\left|\int_{M} \operatorname{tr}(S) d v_{g}\right|}{\left\|H_{S}\right\|_{2 p} V(M)}(1+\varepsilon)
$$

Theorem 6.3. Let $n \geqslant 2$ and $\left(M^{n}, g\right)$ be a connected, oriented and closed Riemannian manifold isometrically immersed into $\mathbb{R}^{n+1}$ by $X$. Assume that $M$ is endowed with a symmetric and divergence-free $(1,1)$-tensor $S$. Assume in addition that $T$ is positive definite, $H_{S}$ is not identically zero and that for some $q>n$ there holds $V(M)\|B\|_{q}^{n} \leqslant A$. Let $p>1$. Then there exists $\varepsilon_{0}=\varepsilon_{0}(n, p, q, A)>0$, $\alpha=\alpha(n, q)$ and $C=C(n, p, q, A)>0$ such that if $\overline{I_{p, \varepsilon}}$ holds with $\varepsilon<\varepsilon_{0}$, then $M$ is diffeomorphic and $C \varepsilon^{\alpha}$-almost-isometric to the sphere $S(\bar{X}, r)$ with $r=\frac{\left|\int_{M} \operatorname{tr}(S) d v_{g}\right|}{\| H_{S}||_{2 p} V(M)}$. That is, there exists a natural diffeomorphism

$$
F:\left(M, d_{1}\right) \rightarrow\left(S(r), d_{2}\right)
$$

we have

$$
\left|d_{2}\left(F\left(x_{1}\right), F\left(x_{2}\right)\right)-d_{1}\left(x_{1}, x_{2}\right)\right| \leqslant C r \varepsilon^{\alpha} \quad \forall x_{1}, x_{2} \in M .
$$

Moreover, $M$ is embedded and $X(M)$ is a starshaped hypersurface.

It is now classical for such pinching results that the proof needs two steps. The first one gives an $L^{2}$-proximity of the hypersurface with the desired sphere using the pinching condition. This step is given by Lemmas 6.4 and 6.5. The second step, which does not depend on any pinching, allows to go from $L^{2}$ to $L^{\infty}$ proximity. We will recall the needed lemmas without proof.

First, we have these two elementary lemmas.

Lemma 6.4. If $I_{p, \varepsilon}$ holds, then

$$
\left\|X^{T}\right\|_{2}^{2} \leqslant \varepsilon\|X\|_{2}^{2}
$$

Proof:

We have

$$
\begin{aligned}
\left\|X^{T}\right\|_{2}^{2} V(M) & =\int_{M}|X|^{2} d v_{g}-\int_{M}\langle X, \nu\rangle^{2} d v_{g} \\
& \leqslant\|X\|_{2}^{2} V(M)-\frac{1}{\left\|H_{S}\right\|_{2 p}^{2}} \int_{M} H_{S}\langle X, \nu\rangle d v_{g} \\
& \leqslant\|X\|_{2}^{2} V(M)-\frac{1}{\left\|H_{S}\right\|_{2 p}^{2}} \int_{M} \operatorname{tr}(S) d v_{g} \\
& \leqslant\|X\|_{2}^{2} V(M)\left(1-\frac{1}{1+\varepsilon}\right) \\
& \leqslant\|X\|_{2}^{2} V(M) \varepsilon
\end{aligned}
$$

Lemma 6.5. If $I_{p, \varepsilon}$ holds, then

$$
1-\frac{\|X\|_{1}}{\|X\|_{2}} \leqslant K(p) \varepsilon
$$


where $K=K(p)$ is a constant depending only on $p$.

Proof: From the pinching condition and th Hsiung-Minkowski formula, we have

$$
\begin{aligned}
\left\|H_{S}\right\|_{2 p}\|X\|_{2} & \leqslant \frac{\int_{M} \operatorname{tr}(S)}{V(M) \sqrt{1-\varepsilon}} \\
& \leqslant \frac{\int_{M}\left|\left\langle X, H_{S}\right\rangle\right|}{V(M) \sqrt{1-\varepsilon}}
\end{aligned}
$$

Now, using the Hölder inequality and the $\log$-convexity of $\|\cdot\|_{q}$ with respect to $q$, we get

$$
\begin{aligned}
\left\|H_{S}\right\|_{2 p}\|X\|_{2} & \leqslant \frac{1}{\sqrt{1-\varepsilon}}\left\|H_{S}\right\|_{2 p}\|X\|_{\frac{2 p}{2 p-1}} \\
& \leqslant \frac{1}{\sqrt{1-\varepsilon}}\left\|H_{S}\right\|_{2 p}\|X\|_{1}^{1-\frac{1}{p}}\|X\|_{2}^{\frac{1}{p}} .
\end{aligned}
$$

Finally, we obtain

$$
\|X\|_{2} \leqslant(1-\varepsilon)^{-\frac{p}{2(p-1)}}\|X\|_{1}
$$

and hence

$$
1-\frac{\|X\|_{1}}{\|X\|_{2}} \leqslant 1-(1-\varepsilon)^{\frac{p}{2(p-1)}} \leqslant K(p) \varepsilon
$$

with $K=K(p)=\frac{p}{2(p-2)}$.

Now, we recall without proof the two following lemmas which are independant of any pinching conditions.

Lemma 6.6. 3, 24 Let $q>n$ be a real number. There exists a constant $\Gamma(n, q)>$ 0 , so that for any isometrically immersed, compact submanifold $M^{n}$ of $\mathbb{R}^{n+1}$ we have

$$
\||X-\bar{X}|-\| X-\bar{X}\left\|_{2}\right\|_{\infty} \leqslant \Gamma\left(V(M)\|H\|_{q}^{n}\right)^{\frac{\gamma}{n}}\|X-\bar{X}\|_{2}\left(1-\frac{\|X-\bar{X}\|_{1}}{\|X-\bar{X}\|_{2}}\right)^{\frac{1}{2(\gamma+1)}}
$$

where $\gamma=\frac{n q}{2(q-n)}$.

Note that this lemma implies in particular that

$$
\left.\||X|\|_{\infty} \leqslant \Gamma\left(V(M)\|H\|_{q}^{n}\right)^{\frac{\gamma}{2}}+1\right)\|X\|_{2}
$$

Lemma 6.7. 34] Let $q>n$ and $X: M \hookrightarrow \mathbb{R}^{n+1}$ be the immersion of a closed hypersurface. Then there exists a constant $\Gamma^{\prime}=\Gamma^{\prime}(n, q)$, such that

$$
\left\|X^{T}\right\|_{\infty} \leqslant \Gamma^{\prime}\left(V(M)\|B\|_{q}^{n}\|X\|_{\infty}\right)^{\frac{\gamma}{\gamma+1}}\left\|X^{T}\right\|_{2}^{\frac{1}{\gamma+1}}
$$

Now, using these lemmas, we can finish in a now classical way the proof of Theorem 6.3. First, combining Lemmas 6.5 and 6.6. we get

$$
\||X|-\| X\left\|_{2}\right\|_{\infty} \leqslant \Gamma A^{\frac{\gamma}{2}} K^{\frac{1}{2(\gamma+1)}}\|X\|_{2} \varepsilon^{\frac{1}{2(\gamma+1)}}
$$


Now, assuming that $\varepsilon<1$, we get from $\frac{\left.I_{p, \varepsilon}\right]}{\text { that }}\|X\|_{2} \leqslant 2 \frac{\left|\int_{M} \operatorname{tr}(S) d v_{g}\right|}{\left\|H_{S}\right\|_{2 p} V(M)}$. For more compactness in the formulas, we will denote $r=\frac{\left|\int_{M} \operatorname{tr}(S) d v_{g}\right|}{|| H_{S} \|_{2_{p}} V(M)}$ and so we have

$$
\|X\|_{2} \leqslant 2 r .
$$

Thus, using $(29),\left(I_{p, \varepsilon}\right)$, and 30 we get

$$
\begin{aligned}
\||X|-r\|_{\infty} & \leqslant\||X|-\| X\left\|_{2}\right\|_{\infty}+\left|\|X\|_{2}-r\right| \\
& \leqslant 2 r \Gamma A^{\frac{\gamma}{2}} K^{\frac{1}{2(\gamma+1)}} \varepsilon^{\frac{1}{2(\gamma+1)}}+r \varepsilon \\
& \leqslant r\left(2 \Gamma A^{\frac{\gamma}{2}} K^{\frac{1}{2(\gamma+1)}} \varepsilon^{\frac{1}{2(\gamma+1)}}+\varepsilon\right) \\
& \leqslant C_{1} r \varepsilon^{\frac{1}{2(\gamma+1)}}
\end{aligned}
$$

where $C_{1}=\left(2 \Gamma A^{\frac{\gamma}{2}} K^{\frac{1}{2(\gamma+1)}}+1\right)$. Note that the last line comes from the fact that $\frac{1}{2(\gamma+1)}<1$ and we have assumed $\varepsilon<1$. We want to point out that the constant $C_{1}$ depends on $n, p, q$ and $A$. We deduce immediately from this that

$$
\|X\|_{\infty} \leqslant r\left(1+C_{1} \varepsilon^{\frac{1}{2(\gamma+1)}}\right) .
$$

On the other hand, using successively Lemma 6.7. Lemma 6.4 (32) and $I_{p, \varepsilon}$ we get

$$
\begin{aligned}
\left\|X^{T}\right\|_{\infty} & \leqslant \Gamma^{\prime}\left(A\|X\|_{\infty}\right)^{\frac{\gamma}{\gamma+1}}\left\|X^{T}\right\|_{2}^{\frac{1}{\gamma+1}} \\
& \leqslant \Gamma^{\prime}\left(A\|X\|_{\infty}\right)^{\frac{\gamma}{\gamma+1}}\|X\|_{2}^{\frac{1}{\gamma+1}} \varepsilon^{\frac{1}{2(\gamma+1)}} \\
& \leqslant \Gamma^{\prime} A^{\frac{\gamma}{2}}\left(\Gamma A^{\frac{\gamma}{2}}+1\right)^{\frac{\gamma}{\gamma+1}}\|X\|_{2} \varepsilon^{\frac{1}{2(\gamma+1)}} \\
& \leqslant 2 r \Gamma^{\prime} A^{\frac{\gamma}{2}}\left(\Gamma A^{\frac{\gamma}{2}}+1\right)^{\frac{\gamma}{\gamma+1}} \varepsilon^{\frac{1}{2(\gamma+1)}} \\
& \leqslant C_{2} r \varepsilon^{\frac{1}{2(\gamma+1)}},
\end{aligned}
$$

where $C_{2}=2 \Gamma^{\prime} A^{\frac{\gamma}{2}}\left(\Gamma A^{\frac{\gamma}{2}}+1\right)^{\frac{\gamma}{\gamma+1}}$ is a constant depending only on $n, p, q$ and $A$.

Now, we set $\varepsilon_{1}=\inf \left\{\frac{\sqrt{2}-1}{\sqrt{2} C_{1}}, \frac{1}{2 C_{2}}\right\}^{2(\gamma+1)}$. Note that $\varepsilon_{1}$ depends only on $n, p, q$ and $A$ and if $\varepsilon \leqslant \varepsilon_{1}$, we have

$$
C_{1} \varepsilon^{\frac{1}{2(\gamma+1)}}<1, \quad C_{2} \varepsilon^{\frac{1}{2(\gamma+1)}}<1 \quad \text { and } \quad\left(1-C_{1} \varepsilon^{\frac{1}{2(\gamma+1)}}\right)^{2}-\left(C_{2} \varepsilon^{\frac{1}{2(\gamma+1)}}\right)^{2} \leqslant \frac{1}{4} .
$$

From this last inequality, with 32 and 33 , we deduce that

$$
\langle X, \nu\rangle^{2}=|X|^{2}-\left|X^{T}\right|^{2} \geqslant \frac{r^{2}}{4} \text {. }
$$

Now, we consider the map

$$
\begin{aligned}
F: M & \longrightarrow S(0, r) \\
x & \longmapsto r \frac{X(x)}{|X(x)|} .
\end{aligned}
$$

First of all, from (31) and the fact that $C_{1} \varepsilon^{\frac{1}{2(\gamma+1)}}<1$, we get $\||X|-r\|_{\infty}<r$. Therefore $|X|$ never vanishes and so $F$ is well defined. Now, we will compute the 
differential of $F$. Let $u \in T_{x} M$ be a unit vector. We get immediately that

$$
d F_{x}(u)=\frac{r}{|X|}\left(u-\frac{\langle u, X\rangle}{|X|^{2}}\right)
$$

and so

$$
\begin{aligned}
\left.|| d F_{x}(u)\right|^{2}-1 \mid & \leqslant\left|\frac{r^{2}}{|X|^{2}}-1\right|+\frac{r^{2}}{|X|^{4}}\langle u, X\rangle^{2} \\
& \leqslant \frac{1}{|X|^{2}}|| X|-r| \cdot|| X|+r|+\frac{r^{2}\left|X^{T}\right|^{2}}{|X|^{4}}
\end{aligned}
$$

Now, from (31), (32), (33) and the fact that $C_{1} \varepsilon^{\frac{1}{2(\gamma+1)}}<1$, we get

$$
\left.|| d F_{x}(u)\right|^{2}-1 \mid \leqslant \frac{2 r^{2} C_{1} \varepsilon^{\frac{1}{2(\gamma+1)}}}{|X|^{2}}+\frac{r^{3} C_{2} \varepsilon^{\frac{1}{2(\gamma+1)}}}{|X|^{3}} .
$$

Finally, from (34), we deduce that $\frac{1}{|X|} \leqslant \frac{2}{r}$ and so

$$
\left.|| d F_{x}(u)\right|^{2}-1 \mid \leqslant \frac{2 C_{1}+C_{2}}{4} \varepsilon^{\frac{1}{2(\gamma+1)}}=C_{3} \varepsilon^{\frac{1}{2(\gamma+1)}}
$$

We put $\varepsilon_{0}=\inf \left\{\varepsilon_{1},\left(\frac{1}{2 C_{3}}\right)^{2(\gamma+1)}\right\}$. We get from this that $\left|d F_{x}(u)\right|^{2} \geqslant \frac{1}{2}$ which implies that $F$ is a local diffeomorphism. Since $M$ and $S(0, r)$ are connected, $M$ is closed and $S(0, r)$ is simply connected, then $F$ is a global diffeomorphism.

In addition, since $F$ which is of the form $G \circ X$ is injective, then $X$ is injective and so the immersion $X$ is an embedding.

Moreover, the condtion (34) and the connectedness of $M$ implies that the support function $\langle X, \nu\rangle$ never vanishes (say is positive if $\nu$ is the outward unit normal) which implies that $X(M)$ is a starshaped hypersurface (see [21]).

Finally, from (35), we get that for any $x, y \in M$

$$
\left|d_{2}(f(x), f(y))-d(x, y)\right| \leqslant M d_{1}(x, y),
$$

where $d_{1}$ and $d_{2}$ are the Riemannian distance on $M$ and $S(0, r)$ respectively and $M=\sup \left\{\left.|| d F x(u)\right|^{2}-1 \mid, x \in M u \in U_{x} M\right\}$. Hence, we deduce that

$$
\left|d_{2}(f(x), f(y))-d(x, y)\right| \leqslant 2 \pi r C_{3} \varepsilon^{\alpha} .
$$

This concludes the proof of Theorem 6.3 by setting $C=2 \pi C_{3}$, which depends onpy on $n, p, q$ and $A$ as $C_{3}$.

Now, we introduce the pinching condition associated with the optimal inequality involving the first eigenvalue $\lambda_{1, T}$ of the operator $L_{T}$, the first eigenavlue $\sigma_{1, T}$ of the generalized Steklov problem associated with $L_{T}$, the first eiganvalue $\Lambda_{1}$ of the Paneitz-like operator $P_{a, b}$ and the extrinsic radius $R(M)$. Namely, for $p>1$, we set

$$
\begin{array}{ll}
\left(L_{p, \varepsilon}\right) & \lambda_{1, T}\left(\int_{M} \operatorname{tr}(S) d v_{g}\right)^{2} \geqslant(1-\varepsilon)\left(\int_{M} \operatorname{tr}(T) d v_{g}\right)\left\|H_{S}\right\|_{2 p}^{2} V(M), \\
\left(S_{p, \varepsilon}\right) & \sigma_{1, T}\left(\int_{M} \operatorname{tr}(S) d v_{g}\right)^{2} \geqslant(1-\varepsilon)\left(\int_{\Omega} \operatorname{tr}(T) d v_{g}\right)\left\|H_{S}\right\|_{2 p}^{2} V(M),
\end{array}
$$




$$
\begin{gathered}
\left(W_{p, \varepsilon}\right) \quad \alpha_{1}\left(\int_{M} \operatorname{tr}(S) d v_{g}\right)^{2} \geqslant(1-\varepsilon)(n V(\Omega)+b(n-1) V(M))\left\|H_{S}\right\|_{2 p}^{2} V(M), \\
\left(B_{p, \varepsilon}\right) \quad \beta_{1}\left(\int_{M} \operatorname{tr}(S) d v_{g}\right)^{2} \geqslant(1-\varepsilon) n \tau V(\Omega)\left\|H_{S}\right\|_{2 p}^{2} V(M) \\
\left(P_{p, \varepsilon}\right) \\
\Lambda_{1}\left(\int_{M} \operatorname{tr}(S) d v_{g}\right)^{2} \geqslant(1-\varepsilon)\left(\int_{M}\left(n^{2} H^{2}+(n a+b) R_{g}\right) d v_{g}\right)\left\|H_{S}\right\|_{2 p}^{2} V(M), \\
\left(R_{p, \varepsilon}\right) \quad R(M)^{2} \leqslant \frac{\left(\int_{M} \operatorname{tr}(S) d v_{g}\right)^{2}}{\left\|H_{S}\right\|_{2 p}^{2} V(M)^{2}}(1+\varepsilon) .
\end{gathered}
$$

Proposition 6.8. Let $p>1$ and $\varepsilon \in\left(0, \frac{1}{2}\right)$. If one of the pinching conditions $\left(L_{p, \varepsilon}\right), S_{p, \varepsilon}, W_{p, \varepsilon}, B_{p, \varepsilon}, P_{p, \varepsilon}$ or $\left(R_{p, \varepsilon}\right)$ holds, then also holds $\left(I_{2,2 \varepsilon}\right)$.

Proof: First, we recall that from the Rayleigh quotient with coordinates as test functions we have

$$
\lambda_{1}\left(L_{T}\right) \int_{M}|X|^{2} d v_{g} \leqslant \int_{M} \operatorname{tr}(T) d v_{g}
$$

Now, if $\left(L_{p, \varepsilon}\right)$ holds, then, we have

$$
\begin{aligned}
\|X\|_{2}^{2} & \leqslant \frac{\int_{M} \operatorname{tr}(T) d v_{g}}{\lambda_{1}\left(L_{T}\right)} \\
& \leqslant \frac{1}{1-\varepsilon} \cdot \frac{\left(\int_{M} \operatorname{tr}(S) d v_{g}\right)^{2}}{\|H\|_{2 p}^{2} V(M)} \\
& \leqslant(1+2 \varepsilon) \frac{\left(\int_{M} \operatorname{tr}(S) d v_{g}\right)^{2}}{\|H\|_{2 p}^{2} V(M)},
\end{aligned}
$$

if $\varepsilon<\frac{1}{2}$, which is exactly $\left(I_{2, \varepsilon}\right)$.

The same argument holds for the other pinching conditions, we will write them down more quickly.

For the Steklov problem, from $(10)$ and if $\left[S_{p, \varepsilon}\right]$ holds for $\varepsilon<\frac{1}{2}$, we have

$$
\|X\|_{2}^{2} \leqslant \frac{\int_{\Omega} \operatorname{tr}(T) d v_{g}}{\sigma_{1, T} V(M)} \leqslant \frac{1}{1-\varepsilon} \cdot \frac{\left(\int_{M} \operatorname{tr}(S) d v_{g}\right)^{2}}{\|H\|_{2 p}^{2} V(M)} \leqslant(1+2 \varepsilon) \frac{\left(\int_{M} \operatorname{tr}(S) d v_{g}\right)^{2}}{\|H\|_{2 p}^{2} V(M)} .
$$

For the Steklov-Wentzell problem, if $\left(W_{p, \varepsilon}\right)$ holds for $\varepsilon<\frac{1}{2}$, we get from (14)

$$
\|X\|_{2}^{2} \leqslant \frac{n V(\Omega)+(n-1) V(M)}{\alpha_{1} V(M)} \leqslant \frac{1}{1-\varepsilon} \cdot \frac{\left(\int_{M} \operatorname{tr}(S) d v_{g}\right)^{2}}{\|H\|_{2 p}^{2} V(M)} \leqslant(1+2 \varepsilon) \frac{\left(\int_{M} \operatorname{tr}(S) d v_{g}\right)^{2}}{\|H\|_{2 p}^{2} V(M)} .
$$

For the biharmonic Steklov problem, we recall that 17 holds, that is,

$$
\beta_{1} \int_{M}\|X\|^{2} d v_{g} \leqslant \int_{\Omega}\left(\|\bar{B}\|^{2}+n \tau\right) d v_{\bar{g}} .
$$

Since $M$ is a hypersurface, $\Omega$ is a domain of $\mathbb{R}^{n}$ and so $\bar{B}=0$, which give

$$
\beta_{1} \int_{M}\|X\|^{2} d v_{g} \leqslant n \tau V(\Omega)
$$


Hence, if $B_{p, \varepsilon}$ holds for $\varepsilon<\frac{1}{2}$, we obtain

$$
\|X\|_{2}^{2} \leqslant \frac{n \tau V(\Omega)}{\beta_{1} V(M)} \leqslant \frac{1}{1-\varepsilon} \cdot \frac{\left(\int_{M} \operatorname{tr}(S) d v_{g}\right)^{2}}{\|H\|_{2 p}^{2} V(M)} \leqslant(1+2 \varepsilon) \frac{\left(\int_{M} \operatorname{tr}(S) d v_{g}\right)^{2}}{\|H\|_{2 p}^{2} V(M)} .
$$

For the Paneitz-like operators, from $(18)$ and if $\left(P_{p, \varepsilon}\right)$ holds for $\varepsilon<\frac{1}{2}$, we get

$$
\|X\|_{2}^{2} \leqslant \frac{\int_{M}\left(n^{2}|H|^{2}+(n a+b) R\right)}{\sigma_{1, T}} \leqslant \frac{1}{1-\varepsilon} \cdot \frac{\left(\int_{M} \operatorname{tr}(S) d v_{g}\right)^{2}}{\|H\|_{2 p}^{2} V(M)} \leqslant(1+2 \varepsilon) \frac{\left(\int_{M} \operatorname{tr}(S) d v_{g}\right)^{2}}{\|H\|_{2 p}^{2} V(M)} .
$$

Finally, for the extrinsic radius, we have obviously

$$
\|X\|_{2}^{2} \leqslant R(M)^{2} \leqslant(1+\varepsilon) \frac{\left(\int_{M} \operatorname{tr}(S) d v_{g}\right)^{2}}{\|H\|_{2 p}^{2} V(M)} .
$$

This concludes the proof of the Proposition. From this proposition and Theorem 6.3 , we obtain these final two corollaries. The first one give a pinching result for the first eigenvalue of the operators $L_{T}$ and Paneitz-like operators andas well as for the extrinsic radius for which the hypersurfaces are not supposed to be embedded.

Corollary 6.9. Let $\left(M^{n}, g\right)$ be a closed, connected and oriented manifold isometrically immersed into the Euclidean space $\mathbb{R}^{n+1}$ by $X$. Let $S$ and $T$ be two symmetric $(1,1)$-tensors with vanishing divergence. Assume moreover that $T$ is positive definite, $H_{S}$ does not vanish identically and that for some $q>n$ there holds $V(M)\|B\|_{q}^{n} \leqslant A$. Let $p>1$. Then there exists $\varepsilon_{0}=\varepsilon_{0}(n, p, q, A)>0$, $\alpha=\alpha(n, q)$ and $C=C(n, p, q, A)>0$ such that if $\left(L_{p, \varepsilon}\right),\left(P_{p, \varepsilon}\right)$ or $\left(R_{p, \varepsilon}\right)$ holds with $\varepsilon<\varepsilon_{0}$, then $M$ is diffeomorphic and $C \varepsilon^{\alpha}$-almost-isometric to the sphere $S(\bar{X}, r)$ with $r=\frac{\left|\int_{M} \operatorname{tr}(S) d v_{g}\right|}{\left\|H_{S}\right\|_{2_{p}} V(M)}$. Moreover, $M$ is embbeded and $X(M)$ is a starshaped hypersurface.

This second corollary concerns the Steklov problems where the hypersurfaces need to be embedded and bound domains.

Corollary 6.10. Let $\left(M^{n}, g\right)$ be a closed, connected and oriented manifold isometrically embedded into the Euclidean space $\mathbb{R}^{n+1}$ by $X$. Let $S$ and $T$ be two symmetric $(1,1)$-tensors with vanishing divergence. Assume moreover that $T$ is positive definite, $H_{S}$ does not vanish identically and that for some $q>n$ there holds $V(M)\|B\|_{q}^{n} \leqslant A$. Let $p>1$. Then there exists $\varepsilon_{0}=\varepsilon_{0}(n, p, q, A)>0$, $\alpha=\alpha(n, q)$ and $C=C(n, p, q, A)>0$ such that if $\left(S_{p, \varepsilon}\right.$, $W_{p, \varepsilon}$ or $B_{p, \varepsilon}$ holds with $\varepsilon<\varepsilon_{0}$, then $M$ is diffeomorphic and $C \varepsilon^{\alpha}$-almost-isometric to the sphere $S(\bar{X}, r)$ with $r=\frac{\left|\int_{M} \operatorname{tr}(S) d v_{g}\right|}{\left\|H_{S}\right\|_{2_{p}} V(M)}$. Moreover $X(M)$ is a starshaped hypersurface.

We finish this paper with the following remarks.

Remarks 6.11. (1) First, we want to recall that the condition (35) allwos to obtain a proximity with the sphere for the Lipschitz distance which implies in particular that the spectrum of the Laplacian is close to the spectrum of the sphere (of corresponding radius).

(2) In our theorems, we consider a control on the second fundamental. This condition is required to get the promixity for the Lipschitz ditance and the 
diffeomorphism. It is known that such a control is necessary. With a control only on the mean curvature, we can only get a proximity in Hausdorff distance ( see [2, 14, 30] for instance).

(3) In 2], some examples of hypersurfaces satisying the pinching condition (I, $)$ with $S=$ Id with a control of the mean curvature but non diffeomorphic to the sphere are given.

(4) Always in 2], the authors show that an asymptotic closeness with the spectrum of the Laplacian with the spectrum of the sphere can be obtained (see also [3] ) with a control of the mean curvature. This result can be adapted here with minor modifications.

\section{REFERENCES}

[1] H. Alencar, M.P. Do Carmo \& H. Rosenberg, On the first eigenvalue of Linearized operator of the r-th mean curvature of a hypersurface, Ann. Glob. Anal. Geom., 11 (1993), 387-395.

[2] E. Aubry \& J.F. Grosjean, Spectrum of hypersurfaces with small extrinsic radius or large $\lambda_{1}$ in Euclidean spaces, J. Funct. Anal. 271 (2016), no 5, 1213-1242.

[3] E. Aubry, J.F. Grosjean \& J. Roth, hypersurfaces with small extrinsic radius or large $\lambda_{1}$ in Euclidean spaces, arXiv:1009.2010.

[4] L.J. Alias \& J.M. Malacarne, On the first eigenvalue of the linearized operator of the higher order mean curvature for closed hypersurfaces in space forms, Illinois J. Math. 48 (1) (2004) 219-240.

[5] G. Auchmuty, Steklov Eigenproblems and the Representation of Solutions of Elliptic Boundary Value Problems, Numer. Funct. Anal. Optim. 25 (2004), no. 3-4, 321-348.

[6] D. Bakry \& M. Émery. Diffusions hypercontractives. In Séminaire de probabilités, XIX, 1983/84, volume 1123 of Lecture Notes in Math., pages 177206. Springer, Berlin, 1985.

[7] J.L.M. Barbosa \& A.G. Colares, Stability of hypersurfaces with constant r-mean curvature, Ann. Global Anal. Geom. 15 (1997), 277-297.

[8] M. Batista, M.P. Cavalcante \& J. Pyo, Some isomperimetric inequalities and eigenvalue estimates in weighted manifolds, J. Math. Anal. Appl., 419(1) (2014), 617-626.

[9] Batista, Santos, The first Stekloff eigenvalue in weighted Riemannian manifolds, arXiv:1504.02630, 2015.

[10] T. Branson, Differential Operators Canonically Associated to a Conformal Structure, Math. Scand. 57 (1985), 293-345.

[11] S. Brendle, Embedded self-similar shrinkers of genus 0, Ann. Math. 183 (2016), 715-728.

[12] D. Buoso \& L. Provenzano, On the eigenvalues of a biharmonic Steklov problem, Integral Methods in Science and Engineering: Theoretical and Computational Advances, Birkhuser, 2015.

[13] D. Chen \& H. Li, The sharp estimates for the first eigenvalue of Paneitz operator on 4dimensional submanifolds, arXiv:1010.3102.

[14] B. Colbois \& J.F. Grosjean, A pinching theorem for the first eigenvalue of the Laplacian on hypersurfaces of Euclidean spaces, Comment. Math. Helv., 82 (2007), 175-195.

[15] M. Dambrine, D. Kateb and J. Lamboley, An extremal eigenvalue problem for the WentzellLaplace operator, Ann. I. H. Poincaré, 33 (2016), no. 2, 409-450.

[16] Z. Djadli, E. Hebey \& M. Ledoux, Paneitz-type operators and applications, Duke Math. J., 104(1) (2000), 129-169.

[17] M.C. Domingo-Juan \& V. Miquel, Reilly's type inequality for the Laplacian associated to a density related with shrinkers for MCF, arXiv:1503.01332.

[18] A. El Soufi \& S. Ilias, Une inégalité de type "Reilly" pour les sous-variéts de l'espace hyperbolique, Comment. Math. Helv. 67(2) (1992), 167-181.

[19] A. El Soufi, E.M. Harrell II, \& S. Ilias, Universal inequalities for the eigenvalues of Laplace and Schrödinger operators on submanifolds, Trans. Amer. Math. Soc. 361(5) (2009), 23372350 . 
[20] J.F. Grosjean, Upper bounds for the first eigenvalue of the Laplacian on compact manifolds, Pac. J. Math. 206(1) (2002), 93-111.

[21] P.F. Guan \& S. Shen, A rigidity theorem for hypersurfaces in higher dimensional space forms, Analysis, complex geometry, and mathematical physics: in honor of Duong H. Phong, 6165, Contemp. Math., 644, Amer. Math. Soc., Providence, RI, 2015

[22] C.C. Hsiung, Some integral formulae for closed hypersurfaces, Math. Scand 2 (1954), 286-294.

[23] G. Huisken, Asymptotic behavior for singularities of the mean curvature flow, J. Differential Geom. 31 (1990), 285-299.

[24] Y.Hu, H. Xu, and E. Zhao, First eigenvalue pinching for Euclidean hypersurfaces via k-th mean curvatures, Ann. Glob. Anal. Geom. (2015), 1-13.

[25] S. Ilias \& O. Makhoul, A Reilly inequality for the first Steklov eigenvalue, Diff. Geom. Appl. 29(5) (2011), 699-708.

[26] A. Lichnerowicz, Variétés riemanniennes à tenseurr $C$ non négatif. C.R. Acad. Sc. Paris Serie A, 271 (1970) A650-A653.

[27] S. Paneitz, A quartic conformally covariant differential operator for arbitrary pseudoRiemannian manifolds, preprint, 1983.

[28] S. Paneitz, A quartic conformally covariant differential operator for arbitrary pseudoRiemannian manifolds, SIGMA 4 (2008), article 036.

[29] R.C. Reilly, On the first eigenvalue of the Laplacian for compact submanifolds of Euclidean space, Comment. Math. Helv. 52 (1977), 525-533.

[30] J. Roth, Extrinsic radius pinching for hypersurfaces of space forms, Diff. Geom.Appl. 25(5) (2007) 485-499.

[31] J. Roth, Extrinsic radius pinching in space forms with nonnegative sectional curvature, Math. Z. 258(1) (2008) 227-240.

[32] J. Roth, Upper bounds for the first eigenvalue of the Laplacian in terms of anisiotropic mean curvatures, Results Math. 64 (3-4) (2013), pp 383-403.

[33] J. Roth, General Reilly-type inequalities for submanifolds of weighted Euclidean spaces, Colloq. Math. 144(1) (2016),127-136.

[34] J. Roth \& J. Scheuer, Pinching of the first eigenvalue for second order operators on hypersurfaces of the Euclidean space, Ann. Glob. Anal. Geom. 51(3) (2017), 287-304.

[35] W. Steklov, Sur les problèmes fondamentaux de la physique mathémnatique (suite et fin), Ann. Sci. École Norm. Sup. (3) 319 (1902), 455-490.

[36] G. Wei \& W. Wylie, Comparison Geometry for the Bakry-Émery Ricci curvature, J. Diff. Geom, 83 (2009), 377-405.

[37] C. Xia \& Q. Wang, Eigenvalues of the Wentzell-Laplace Operator and of the Fourth Order Steklov Problems, arXiv:1506.03780.

[38] P. Yang \& X. Xu, Positivity of Paneitz operators, Discrete Cont. Dyn. Syst. 7(2) (2001), 329342.

Laboratoire d'Analyse et de Mathématiques Appliquées, UPEM-UPEC, CNRS, F-77454 MARNE-LA-VALLÉE

E-mail address: julien.roth@u-pem.fr 